$11-1-2013$

\title{
On Bayesian Estimation and Predictions for Two- Component Mixture of the Gompertz Distribution
}

Navid Feroze

Allama Iqbal Open University, Islamabad, Pakistan, navidferoz@hotmail.com

Muhammad Aslam

Quaid-i-Azam University, Islamabad, Pakistan, aslamsdqu@yahoo.com

Follow this and additional works at: http://digitalcommons.wayne.edu/jmasm

Part of the Applied Statistics Commons, Social and Behavioral Sciences Commons, and the Statistical Theory Commons

\section{Recommended Citation}

Feroze, Navid and Aslam, Muhammad (2013) "On Bayesian Estimation and Predictions for Two-Component Mixture of the Gompertz Distribution," Journal of Modern Applied Statistical Methods: Vol. 12 : Iss. 2 , Article 16.

DOI: $10.22237 /$ jmasm/1383279300

Available at: http://digitalcommons.wayne.edu/jmasm/vol12/iss2/16

This Regular Article is brought to you for free and open access by the Open Access Journals at DigitalCommons@WayneState. It has been accepted for inclusion in Journal of Modern Applied Statistical Methods by an authorized editor of DigitalCommons@WayneState. 


\title{
On Bayesian Estimation and Predictions for Two-Component Mixture of the Gompertz Distribution
}

\author{
Navid Feroze \\ Allama Iqbal Open University \\ Islamabad, Pakistan
}

\author{
Muhammad Aslam \\ Quaid-i-Azam University \\ Islamabad, Pakistan
}

\begin{abstract}
Mixtures models have received sizeable attention from analysts in the recent years. Some work on Bayesian estimation of the parameters of mixture models have appeared. However, the were restricted to the Bayes point estimation The methodology for the Bayesian interval estimation of the parameters for said models is still to be explored. This paper proposes the posterior interval estimation (along with point estimation) for the parameters of a two-component mixture of the Gompertz distribution. The posterior predictive intervals are also derived and evaluated. Different informative and noninformative priors are assumed under a couple of loss functions for the posterior analysis. A simulation study was carried out in order to make comparisons among different point and interval estimators. The applicability of the results is illustrated via a real life example.
\end{abstract}

Keywords: $\quad$ Bayes estimators, loss functions, posterior distributions, censoring, mixture densities

\section{Introduction}

The Gompertz distribution is used to model survival times, human mortality and actuarial tables. It has many real life applications, especially in medical and actuarial studies. The Gompertz distribution is also used as a survival model in reliability. It has an increasing hazard rate for the life of the systems. Due to its complicated form it has not received enough attention in past. However, recently, this distribution has received considerable attention from demographers and actuaries. Pollard and Valkovics (1992) were the first to deal with the Gompertz distribution thoroughly. However, their results are true only in cases where the initial level of mortality is very close to zero. Willemse and Koppelaar (2000)

Navid Feroze is a lecturer at the Government Post Graduate College Muzaffarābād, Azad Kashmir, Pakistan.Email him at: navidferoz@hotmail.com.Dr. Aslam is a professor in the Department of Statistics. Email him at aslamsdqu@yahoo.com. 
reformulated the Gompertz force of mortality and derived relationships for this new formulation. Jaheen (2003) applied the Bayesian approach on record values from the Gompertz distribution. The simulation study was used for illustration of the results. Wu et al. (2003) derived the point and interval estimators for parameters of the Gompertz distribution under progressive type II censored samples. Wu et al. (2004) used the least square method to estimate the parameters of the Gompertz distribution. Wu et al. (2006) obtained the maximum likelihood estimators and the estimated expected test time for the two-parameter Gompertz distribution under progressive censoring with binomial removals. Khedhair and Gohary (2008) proposed the bivariate Gompertz distribution and completed the analysis for the mixture of components of proposed distribution. Saracoglu et al. (2009) compared the maximum likelihood, uniformly minimum variance unbiased, and Bayes estimators for the parameter of the Gompertz distribution. The numerical example was used for illustration. Ismail (2010) considered the Gompertz distribution as a lifetime model for applying the Bayesian approach to the estimation problem in the case of step stress partially accelerated life tests with two stress levels and type-I censoring. Ismail (2011) discussed the point and interval estimations of a two-parameter Gompertz distribution under partially accelerated life tests with Type-II censoring. Kiani et al. (2012) studied the performance of the Gompertz model with time-dependent covariate in the presence of right censored data. Moreover, the performance of the model was compared at different censoring proportions $(C P)$ and sample sizes.

The mixture models have received great interest from analysts in recent era. These models include finite and infinite numbers of components that can analyze different datasets. A finite mixture of probability distribution is suitable to study a population categorized in number of subpopulations. A population of lifetimes of certain electrical elements can be classified into a number of subpopulations based on causes of failures. The analysis of mixture models under Bayesian framework has developed a significant interest among statisticians. Authors dealing with the Bayesian analysis of mixture models include: Saleem and Aslam (2008), Saleem et al. (2010), Majeed and Aslam (2012) and Kazmi et al. (2012). These contributions are concerned with Bayes point estimation of the parameters. The interval estimation of the parameters of the mixture models under a Bayesian framework has not yet been discussed by any author. We considered point and interval estimation of the parameters for a two-component mixture of the Gompertz distribution. The population of certain items is assumed to be partitioned into two subpopulations. The randomly selected observations from said population are considered to be a part of one of the above mentioned 


\section{FEROZE \& ASLAM}

subpopulations. These subpopulations are assumed to follow the Gompertz distribution. Therefore, the two components mixture of the Gompertz distribution has been proposed to model this population. The observations have been assumed to be right censored. The inverse transformation technique of simulation under a probabilistic mixing has been used to generate data and to evaluate the performance of different estimators.

\section{The Population and the Model}

A density function for the mixture of two component densities with mixing weights $(p, q)$ is

$$
f(x)=p f_{1}(x)+q f_{2}(x), 0<p<1
$$

The following Gompertz distribution is considered for both mixture densities:

$$
f\left(x_{i} ; \alpha_{i}\right)=\alpha_{i} e^{x_{i}-\alpha_{i}\left(e^{x_{i}}-1\right)}, x_{i}>0, \alpha_{i}>0
$$

with the cumulative distribution function as

$$
F\left(x_{i} ; \alpha_{i}\right)=1-e^{-\alpha_{i}\left(e^{x_{i}}-1\right)}
$$

The cumulative distribution function for the mixture model is

$$
F(x)=p F_{1}(x)+q F_{2}(x)
$$

Suppose $n$ items are put on a life testing experiment and $w$ units failed until time $T$, while $n-w$ units are still working. Now based on causes of failure, the failed items are assumed to come either from subpopulation 1 or from subpopulation 2. Therefore it can be observed that $w_{1}$ and $w_{2}$ failed items come from the first and second subpopulation respectively, where $w=w_{1}+w_{2}$. The remaining $n-w$ items are assumed to be censored observations. The likelihood function for above type I censored data can be obtained as 


$$
\begin{aligned}
L\left(\alpha_{1}, \alpha_{2}, p \mid \underline{x}\right) & \propto \prod_{j=1}^{w_{1}}\left\{p f_{1}\left(x_{1 j}\right)\right\} \prod_{j=1}^{w_{2}}\left\{p f_{2}\left(x_{2 j}\right)\right\} \\
& \times[1-F(t)]^{n-w}
\end{aligned}
$$

After simplifications the likelihood function becomes

$$
\begin{aligned}
L\left(\alpha_{1}, \alpha_{2}, p \mid \underline{x}\right) & \propto \sum_{k=0}^{n-w}\left(\begin{array}{c}
n-w \\
k
\end{array}\right) \alpha_{1}^{w_{1}} \alpha_{2}^{w_{2}} p^{n-k-w_{2}} \\
& \times q^{w_{2}+k} e^{-\alpha_{1} \xi_{k}(x)} e^{-\alpha_{2} \xi_{2 k}(x)}
\end{aligned}
$$

where

$$
\xi_{1 k}(x)=\sum_{j=1}^{w_{1}}\left(e^{x_{1 j}}-1\right)+(n-w-k)\left(e^{t}-1\right)
$$

and

$$
\xi_{2 k}(x)=\sum_{j=1}^{w_{2}}\left(e^{x_{2 j}}-1\right)+k\left(e^{t}-1\right)
$$

\section{The Posterior Distributions under Different Priors}

The main difference between the Bayesian and classical inference is the use of prior information under the Bayesian framework. However, in cases where the sufficient prior information regarding the parameter is not available, the use of non-informative priors becomes mandatory. An important non-informative prior, proposed by Laplace (1812), is a uniform prior. It has been applied to many problems, and often the results are entirely satisfactory. Here, this prior has been used for the posterior estimation.

Let $\quad \alpha_{1} \in$ Uniform $\forall \alpha_{1} \in(0, \infty), \quad \alpha_{2} \in$ Uniform $\forall \alpha_{2} \in(0, \infty), \quad$ and $p \sim U(0,1)$. Assuming independence, these priors result into a joint prior that is proportional to a constant. That joint prior has been used to derive the joint posterior distribution of $\alpha_{1}, \alpha_{2}$ and $p$. The marginal distribution for each 


\section{FEROZE \& ASLAM}

parameter can be obtained by integrating the joint posterior distribution with respect to nuisance parameters. The joint posterior distribution is

$$
p\left(\alpha_{1}, \alpha_{2}, p \mid \underline{x}\right)=\frac{\sum_{k=0}^{n-w}\left(\begin{array}{c}
n-w \\
k
\end{array}\right) \alpha_{1}^{w_{1}} \alpha_{2}^{w_{2}} p^{n-k-w_{2}} q^{w_{2}+k} e^{-\alpha_{1} \xi_{1 k}(x)} e^{-\alpha_{2} \xi_{2 k}(x)}}{\sum_{k=0}^{n-w}\left(\begin{array}{c}
n-w \\
k
\end{array}\right)}, \frac{\Gamma\left(\psi_{1 k}, \psi_{2 k}\right) \frac{\Gamma\left(w_{1}+1\right)}{\left\{\xi_{1 k}(x)\right\}^{w_{1}+1}} \frac{\Gamma\left(w_{2}+1\right)}{\left\{\xi_{2 k}(x)\right\}^{w_{2}+1}}}{a_{1}, a_{2}>0}
$$

where $\psi_{1 k}=n-k-w_{2}+1, \psi_{2 k}=w_{2}+k+1$ and $B\left(\psi_{1 k}, \psi_{2 k}\right)$ is standard beta function.

Another non-informative prior has been suggested by Jeffreys (1961), and is frequently used in situations where one does not have much information about the parameters. This prior is defined as

$$
p(\underline{\alpha}) \propto\{|I(\underline{\alpha})|\}^{\frac{1}{2}}
$$

where $f_{i}\left(x_{i} \mid \alpha_{i}\right)$ have been defined in (2) and $p \sim U(0,1)$. Assuming independence, the joint prior is obtained as

$$
h\left(\alpha_{1}, \alpha_{2}, p\right) \propto \frac{1}{\alpha_{1} \alpha_{2}}, a_{1}, a_{2}>0
$$

The joint posterior distribution using the above prior is 
ON PREDICTIONS FOR MIXTURE OF THE GOMPERTZ DISTRIBUTION

$$
p\left(\alpha_{1}, \alpha_{2}, p \mid \underline{x}\right)=\frac{\sum_{k=0}^{n-w}\left(\begin{array}{c}
n-w \\
k
\end{array}\right) \alpha_{1}^{w_{1}-1} \alpha_{2}^{w_{2}-1} p^{n-k-w_{2}} q^{w_{2}+k} e^{-\alpha_{1} \xi_{1 k}(x)} e^{-\alpha_{2} \xi_{2 k}(x)}}{\sum_{k=0}^{n-w}\left(\begin{array}{c}
n-w \\
k
\end{array}\right) B\left(\psi_{1 k}, \psi_{2 k}\right) \frac{\Gamma\left(w_{1}\right)}{\left\{\xi_{1 k}(x)\right\}^{w_{1}}} \frac{\Gamma\left(w_{2}\right)}{\left\{\xi_{2 k}(x)\right\}^{w_{2}}}},
$$

The utilization of informative prior is of much importance under Bayesian inference. The results under informative priors are often better than noninformative priors. The gamma, chi square and exponential priors have been assumed for the posterior analysis in the current study. The combined priors have been obtained by assuming the independence.

Let $\alpha_{1}$ : $\operatorname{Gamma}\left(\sigma_{1}, \tau_{1}\right), \alpha_{2} \sim \operatorname{Gamma}\left(\sigma_{2}, \tau_{2}\right)$ and $p \sim \operatorname{Uniform}(0,1)$. Under the assumption of independence, the joint prior becomes

$$
h\left(\alpha_{1}, \alpha_{2}, p\right) \propto \alpha_{1}^{\sigma_{1}-1} \alpha_{2}^{\sigma_{2}-1} e^{-\left(\alpha_{1} \tau_{1}+\alpha_{2} \tau_{2}\right)}, a_{1}, a_{2}>0
$$

The posterior distribution under the assumption of above prior is

$$
\begin{aligned}
& p\left(\alpha_{1}, \alpha_{2}, p \mid \underline{x}\right)=\frac{\sum_{k=0}^{n-w}\left(\begin{array}{c}
n-w \\
k
\end{array}\right) \alpha_{1}^{w_{1}+\sigma_{1}-1} \alpha_{2}^{w_{2}+\sigma_{2}-1} p^{n-k-w_{2}} q^{w_{2}+k} e^{-\alpha_{1}\left\{\xi_{1 k}(x)+\tau_{1}\right\}} e^{-\alpha_{2}\left\{\xi_{2 k}(x)+\tau_{2}\right\}}}{\sum_{k=0}^{n-w}\left(\begin{array}{c}
n-w \\
k
\end{array}\right) B\left(\psi_{1 k}, \psi_{2 k}\right) \frac{\Gamma\left(w_{1}+\sigma_{1}\right)}{\left\{\xi_{1 k}(x)+\tau_{1}\right\}^{w_{1}+\sigma_{1}}} \frac{\Gamma\left(w_{2}+\sigma_{2}\right)}{\left\{\xi_{2 k}(x)+\tau_{2}\right\}^{w_{2}+\sigma_{2}}}}, \\
& a_{1}, a_{2}>0
\end{aligned}
$$

Again, suppose $\alpha_{1} \sim$ Chi Square $\left(v_{1}\right), \alpha_{2} \sim \operatorname{Chi} \operatorname{Square}\left(v_{2}\right)$ and $p \sim$ Uniform $(0,1)$. Assuming independence, the joint prior becomes

$$
h\left(\alpha_{1}, \alpha_{2}, p\right) \propto \alpha_{1}^{\frac{v_{1}}{2}-1} \alpha_{2}^{\frac{v_{2}}{2}-1} e^{-\frac{\left(\alpha_{1}+\alpha_{2}\right)}{2}}, a_{1}, a_{2}>0
$$

The posterior distribution under the assumption of the prior given in (12) is 


$$
p\left(\alpha_{1}, \alpha_{2}, p \mid \underline{x}\right)=\frac{\sum_{k=0}^{n-w}\left(\begin{array}{c}
n-w \\
k
\end{array}\right) \alpha_{1}^{w_{1}+0.5 v_{1}-1} \alpha_{2}^{w_{2}+0.5 v_{2}-1} p^{n-k-w_{2}} q^{w_{2}+k} e^{-\alpha_{1}\left\{\xi_{1 k}(x)+0.5\right\}} e^{-\alpha_{2}\left\{\xi_{2 k}(x)+0.5\right\}}}{\sum_{k=0}^{n-w}\left(\begin{array}{c}
n-w \\
k
\end{array}\right) B\left(\psi_{1 k}, \psi_{2 k}\right) \frac{\Gamma\left(w_{1}+0.5 v_{1}\right)}{\left\{\xi_{1 k}(x)+0.5\right\}^{w_{1}+0.5 v_{1}}} \frac{\Gamma\left(w_{2}+0.5 v_{2}\right)}{\left\{\xi_{2 k}(x)+0.5\right\}^{w_{2}+0.5 v_{2}}}},
$$

Further, consider $\alpha_{1} \sim \operatorname{Exponential}\left(\varphi_{1}\right), \alpha_{2} \sim \operatorname{Exponential}\left(\varphi_{2}\right) \quad$ and $p \sim \operatorname{Uniform}(0,1)$. Under the assumption of independence, the joint prior becomes

$$
h\left(\alpha_{1}, \alpha_{2}, p\right) \propto e^{-\left(\alpha_{1} \varphi_{1}+\alpha_{2} \varphi_{2}\right)}, a_{1}, a_{2}>0
$$

The posterior distribution under the assumption of above prior is

$$
p\left(\alpha_{1}, \alpha_{2}, p \mid \underline{x}\right)=\frac{\sum_{k=0}^{n-w}\left(\begin{array}{c}
n-w \\
k
\end{array}\right) \alpha_{1}^{w_{1}} \alpha_{2}^{w_{2}} p^{n-k-w_{2}} q^{w_{2}+k} e^{-\alpha_{1}\left\{\xi_{1 k}(x)+\varphi_{1}\right\}} e^{-\alpha_{2}\left\{\xi_{2 k}(x)+\varphi_{2}\right\}}}{\sum_{k=0}^{n-w}\left(\begin{array}{c}
n-w \\
k
\end{array}\right) B\left(\psi_{1 k}, \psi_{2 k}\right) \frac{\Gamma\left(w_{1}+1\right)}{\left\{\xi_{1 k}(x)+\varphi_{1}\right\}^{w_{1}+1}} \frac{\Gamma\left(w_{2}+1\right)}{\left\{\xi_{2 k}(x)+\varphi_{2}\right\}^{w_{2}+1}}},
$$

\section{Bayes Estimators and Posterior Risks}

The Bayes estimators and associated posterior risks have been derived under the squared error loss function (SELF) and precautionary loss function $(P L F)$. The respective expressions have been presented in the following.

Bayes estimator and posterior risk for $\alpha_{1}, \alpha_{2}$ and $p$ under uniform prior using SELF are: 


$$
\begin{aligned}
(B . E)_{P L F}= & {\left[\frac{\sum_{k=0}^{n-w}\left(\begin{array}{c}
n-w \\
k
\end{array}\right) B\left(\psi_{1 k}+2 h, \psi_{2 k}\right) \frac{\Gamma\left(w_{1}+1+2 i\right)}{\left\{\xi_{1 k}(x)\right\}^{w_{1}+1+2 i}} \frac{\Gamma\left(w_{2}+1+2 j\right)}{\left\{\xi_{2 k}(x)\right\}^{w_{2}+1+2 j}}}{\sum_{k=0}^{n-w}\left(\begin{array}{c}
n-w \\
k
\end{array}\right) B\left(\psi_{1 k}, \psi_{2 k}\right) \frac{\Gamma\left(w_{1}+1\right)}{\left\{\xi_{1 k}(x)\right\}^{w_{1}+1}} \frac{\Gamma\left(w_{2}+1\right)}{\left\{\xi_{2 k}(x)\right\}^{w_{2}+1}}}\right] } \\
\rho\left\{(B . E)_{P L F}\right\}= & 2\left[\begin{array}{l}
\sum_{k=0}^{\frac{1}{2}}\left(\begin{array}{c}
n-w \\
k
\end{array}\right) B\left(\psi_{1 k}+2 h, \psi_{2 k}\right) \frac{\Gamma\left(w_{1}+1+2 i\right)}{\left\{\xi_{1 k}(x)\right\}^{w_{1}+1+2 i}} \frac{\Gamma\left(w_{2}+1+2 j\right)}{\left\{\xi_{2 k}(x)\right\}^{w_{2}+1+2 j}} \\
\sum_{k=0}^{n-w}(n-w) B\left(\psi_{1 k}, \psi_{2 k}\right) \frac{\Gamma\left(w_{1}+1\right)}{\left\{\xi_{1 k}(x)\right\}^{w_{1}+1}} \frac{\Gamma\left(w_{2}+1\right)}{\left\{\xi_{2 k}(x)\right\}^{w_{2}+1}} \\
k
\end{array}\right]^{\frac{1}{2}} \\
& -\frac{2 \sum_{k=0}^{n-w}(n-w) B\left(\psi_{1 k}+h, \psi_{2 k}\right) \frac{\Gamma\left(w_{1}+1+i\right)}{\left\{\xi_{1 k}(x)\right\}^{w_{1}+1+i}} \frac{\Gamma\left(w_{2}+1+j\right)}{\left\{\xi_{2 k}(x)\right\}^{w_{2}+1+j}}}{\sum_{k=0}^{n-w}(n-w) B\left(\psi_{1 k}, \psi_{2 k}\right) \frac{\Gamma\left(w_{1}+1\right)}{\left\{\xi_{1 k}(x)\right\}^{w_{1}+1}} \frac{\Gamma\left(w_{2}+1\right)}{\left\{\xi_{2 k}(x)\right\}^{w_{2}+1}}}
\end{aligned}
$$

where, $(B . E)_{P L F}$ and $\rho\left\{(B . E)_{P L F}\right\}$ are the Bayes estimator and the posterior risk under PLF. The Bayes estimates and corresponding risks under other priors can be derived in the similar manner.

\section{Credible intervals}

The credible interval is defined as: Let $g(\alpha \mid \mathrm{x})$ be the posterior distribution then a $100(1-k) \%$ credible interval in any set $C$ is such that $P_{g(\alpha \mid \mathrm{x})}(C)=1-k$. According to Eberly and Casella (2003) the credible interval can also be defined as: $\int_{0}^{L} g(\alpha \mid \mathrm{X}) d \alpha=\frac{k}{2}, \int_{U}^{\infty} g(\alpha \mid \mathrm{x}) d \alpha=\frac{k}{2}$ where $\mathrm{L}$ and $\mathrm{U}$ are the lower and upper limits of the credible interval respectively and $k$ is level of significance. 


\section{FEROZE \& ASLAM}

The $100(1-k) \%$ credible intervals for $\alpha_{1}, \alpha_{2}$ and $p$ under uniform prior can be obtained by solving the following two equations.

$$
\begin{aligned}
& \frac{\sum_{k=0}^{n-w}\left(\begin{array}{c}
n-w \\
k
\end{array}\right) B\left(L^{h}, \psi_{1 k}, \psi_{2 k}\right) \frac{\Gamma\left(w_{1}+1, i L \xi_{1 k}(x)\right) \Gamma\left(w_{2}+1, j L \xi_{2 k}(x)\right)}{\left\{\xi_{1 k}(x)\right\}^{w_{1}+1}\left\{\xi_{2 k}(x)\right\}^{w_{2}+1}}}{\sum_{k=0}^{n-w}\left(\begin{array}{c}
n-w \\
k
\end{array}\right) B\left(\psi_{1 k}, \psi_{2 k}\right) \frac{\Gamma\left(w_{1}+1\right) \Gamma\left(w_{2}+1\right)}{\left\{\xi_{1 k}(x)\right\}^{w_{1}+1}\left\{\xi_{2 k}(x)\right\}^{w_{2}+1}}}=1-\frac{k}{2} \\
& \frac{\sum_{k=0}^{n-w}\left(\begin{array}{c}
n-w \\
k
\end{array}\right) B\left(U^{h}, \psi_{1 k}, \psi_{2 k}\right) \frac{\Gamma\left(w_{1}+1, i U \xi_{1 k}(x)\right) \Gamma\left(w_{2}+1, j U \xi_{2 k}(x)\right)}{\left\{\xi_{1 k}(x)\right\}^{w_{1}+1}\left\{\xi_{2 k}(x)\right\}^{w_{2}+1}}}{\sum_{k=0}^{n-w}\left(\begin{array}{c}
n-w \\
k
\end{array}\right) B\left(\psi_{1 k}, \psi_{2 k}\right) \frac{\Gamma\left(w_{1}+1\right) \Gamma\left(w_{2}+1\right)}{\left\{\xi_{1 k}(x)\right\}^{w_{1}+1}\left\{\xi_{2 k}(x)\right\}^{w_{2}+1}}}=\frac{k}{2}
\end{aligned}
$$

where $h, i, j=0.1, \Gamma(x, y)$ is incomplete gamma function, $B(x, y, z)$ is incomplete beta function and $(L, U)$ define the limits of the credible intervals. Now, the credible interval for $\alpha_{1}, \alpha_{2}$ and $p$ can be derived by putting $h=0, i=1, j=0, h=0, i=0, j=1$, and $h=1, i=0, j=0$, respectively, in the above equations. It should be noted that $\Gamma(x, 0)=\Gamma(x)$ and $B(1, y, z)=B(y, z)$. It can be observed that the explicit solution of the limits for the credible intervals cannot be obtained. The numerical methods have been used to find the approximate solution of the limits.

\section{Posterior Predictive Distributions and Intervals}

The posterior predictive distribution is used to make predictions of future observations, based on the inferences drawn from the data at hand. Posterior predictive distribution can be simply obtained by the product of the posterior distribution and (conditional) independence (given the parameters) of the new observation from the current sample. It can be defined as

$$
\begin{aligned}
g(y \mid \mathrm{x}) & =\int_{0}^{\infty} \int_{0}^{\infty} \int_{0}^{1} p\left(\alpha_{1}, \alpha_{2}, p \mid \mathrm{x}\right) \\
& \times f\left(y ; \alpha_{1}, \alpha_{2}, p\right) d p d \alpha_{1} d \alpha_{2}
\end{aligned}
$$


where $\mathrm{y}=x_{n+1}$ is the future observation given the sample information $x=x_{1}, x_{2}, \ldots$, $x_{n}$, from the model (7). The posterior predictive distribution using (7) and (16) can be obtained as

$$
g(y \mid \mathrm{X})=\frac{\sum_{k=0}^{n-w}\left(\begin{array}{c}
n-w \\
k
\end{array}\right)\left[\frac{B\left(\psi_{1 k}+1, \psi_{2 k}\right)\left(w_{1}+1\right) e^{y}}{\left\{\xi_{1 k}(x)-\ln \left(e^{y}-1\right)\right\}^{w_{1}+2}\left\{\xi_{2 k}(x)\right\}^{w_{2}+1}}+\frac{B\left(\psi_{1 k}, \psi_{2 k}+1\right)\left(w_{2}+1\right) e^{y}}{\left\{\xi_{1 k}(x)\right\}^{w_{1}+1}\left\{\xi_{2 k}(x)-\ln \left(e^{y}-1\right)\right\}^{w_{2}+2}}\right]}{\sum_{k=0}^{n-w}\left(\begin{array}{c}
n-w \\
k
\end{array}\right) \frac{B\left(\psi_{1 k}, \psi_{2 k}\right)}{\left\{\xi_{1 k}(x)\right\}^{w_{1}+1}\left\{\xi_{2 k}(x)\right\}^{w_{2}+1}}}
$$

The posterior predictive interval can be obtained by solving the following two equations

$$
\int_{0}^{L} g(y \mid \mathrm{X}) d y=\frac{\alpha}{2}, \int_{U}^{\infty} g(y \mid \mathrm{x}) d y=\frac{\alpha}{2}
$$

The simplification of the above equations leads to the following equations

$$
\begin{aligned}
& \frac{\sum_{k=0}^{n-w}\left(\begin{array}{c}
n-w \\
k
\end{array}\right)\left[\frac{B\left(\psi_{1 k}+1, \psi_{2 k}\right)}{\left\{\xi_{2 k}(x)\right\}^{w_{2}+1}}\left[\left\{\xi_{1 k}(x)\right\}^{-w_{1}-1}-\left\{\xi_{1 k}(x)-\left(e^{L}-1\right)\right\}^{-w_{1}-1}\right]+\frac{B\left(\psi_{1 k}, \psi_{2 k}+1\right)}{\left\{\xi_{1 k}(x)\right\}^{w_{1}+1}}\left[\left\{\xi_{2 k}(x)\right\}^{-w_{2}-1}-\left\{\xi_{2 k}(x)-\left(e^{L}-1\right)\right\}^{-w_{2}-1}\right]\right]}{\sum_{k=0}^{n-w}\left(\begin{array}{c}
n-w \\
k
\end{array}\right) \frac{B\left(\psi_{1 k}, \psi_{2 k}\right)}{\left\{\xi_{1 k}(x)\right\}^{w_{1}+1}\left\{\xi_{2 k}(x)\right\}^{w_{2}+1}}}=\frac{k}{2}
\end{aligned}
$$

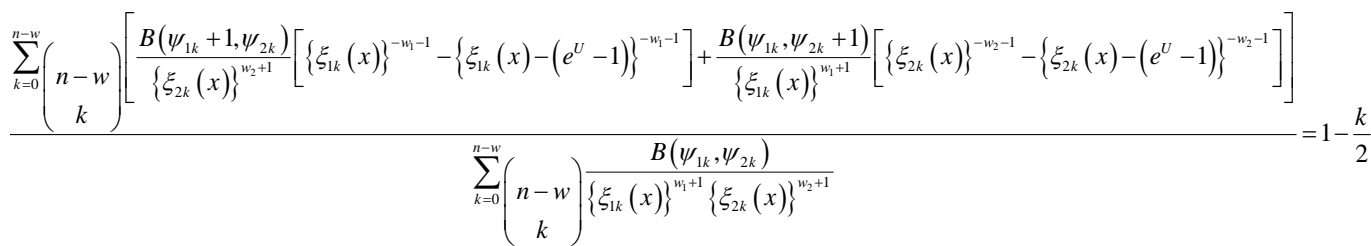

As the limits of the posterior predictive interval cannot be derived explicitly, the numerical solutions of the limits have been obtained by iterative methods. 


\section{Prior Elicitation}

The elicitation is a technique to formulate an expert's knowledge or belief about a certain quantity into a joint probability distribution. In the case of Bayesian analysis, it can be considered as a method to specify the values of hyperparameters in a prior distribution for one or more parameters of the sampling distribution.

Much of the literature on elicitation has been concerned with formulating a probability distribution for unsure quantities when there is no data with which to supplement the knowledge expressed in that distribution. This process facilitates decision-making, where uncertainty about certain phenomena needs to be described in terms of a probability distribution in order to derive the posterior distributions.

To achieve accurate elicitation is a difficult task, even if we are interested in elicitation of a single event. In such a situation, a single probability is needed, but the expert may not be familiar with the concept of probabilities. Even when the expert is familiar with the concept of probabilities, it is by no means straightforward to evaluate a probability value for an event exactly. In such cases, elicitation encourages the expert and the facilitator to consider the meaning of the parameters being elicited. This has two helpful consequences. First, it brings the analysis closer to the application by demanding attention to what is being modeled, and what is reasonable to believe about it. Second, it helps to make the posterior distributions, once calculated, into meaningful quantities. Many methods of elicitation have been discussed in the literature; among those, the method suggested by Aslam (2003) has been used to elicit the prior distribution in the recent study. This method requires the derivation of prior predictive distribution for elicitation. The prior predictive distribution can be defined as

$$
g(y)=\int_{0}^{\infty} \int_{0}^{\infty} \int_{0}^{1} h\left(\alpha_{1}, \alpha_{2}, p\right) f\left(y \mid \alpha_{1}, \alpha_{2}, p\right) d p d \alpha_{1} d \alpha_{2}
$$

where $h\left(\alpha_{1}, \alpha_{2}, p\right)$ and $f\left(y \mid \alpha_{1}, \alpha_{2}, p\right)$ are prior distribution and mixture Gompertz model respectively.

According to (18), the prior predictive distribution under gamma prior is 
ON PREDICTIONS FOR MIXTURE OF THE GOMPERTZ DISTRIBUTION

$g(y)=\frac{\tau_{1}^{\sigma_{1}} \tau_{2}^{\sigma_{2}} e^{y}}{2 \Gamma\left(\sigma_{1}\right) \Gamma\left(\sigma_{2}\right)}\left[\frac{\Gamma\left(\sigma_{1}+1\right) \Gamma\left(\sigma_{2}\right)}{\left(e^{y}+\tau_{1}-1\right)^{\sigma_{1}+1}\left(e^{y}-1\right)^{\sigma_{2}}}+\frac{\Gamma\left(\sigma_{1}\right) \Gamma\left(\sigma_{2}+1\right)}{\left(e^{y}-1\right)^{\sigma_{1}}\left(e^{y}+\tau_{2}-1\right)^{\sigma_{2}+1}}\right]$

In order to elicit the four hyper-parameters, the following four integrals have been considered. The expert's probabilities have been assumed to be 0.15 for each integral:

$$
\int_{1}^{20} g(y)=0.15 \int_{21}^{40} g(y)=0.15 \int_{41}^{60} g(y)=0.15 \quad \int_{61}^{80} g(y)=0.15 \text { and }
$$

A program has been developed in SAS package using the "PROC SYSLIN" command to solve the above integrals simultaneously. The set of hyperparameters with minimum values has been chosen to be the elicited values of the hyper-parameters. These elicited values of the hyper-parameters have been found to be $\left(\sigma_{1}, \tau_{1}, \sigma_{2}, \tau_{2}\right)=(0.000233,0.190642,0.000101,0.189112)$. The prior predictive distribution under chi square prior, given in (12), has been derived as

$$
g(y)=\frac{e^{y}}{2^{0.5\left(v_{1}+v_{2}\right)+1} \Gamma\left(0.5 v_{1}\right) \Gamma\left(0.5 v_{2}\right)}\left[\frac{\Gamma\left(0.5 v_{1}+1\right) \Gamma\left(0.5 v_{2}\right)}{\left(e^{y}-0.5\right)^{0.5 y_{1}+1}\left(e^{y}-1\right)^{0.5 v_{2}}}+\frac{\Gamma\left(0.5 v_{1}\right) \Gamma\left(0.5 v_{2}+1\right)}{\left(e^{y}-1\right)^{0.5 v_{1}}\left(e^{y}-0.5\right)^{0.5 v_{2}+1}}\right]
$$

Using the similar program mentioned above, the elicited values of the hyperparameters are $\left(v_{1}, v_{2}\right)=(1.226759,1.064564)$.

The prior predictive distribution under chi square prior, given in (14), has been presented as

$$
g(y)=0.5 \varphi_{1} \varphi_{2} e^{y}\left(e^{y}-1\right)^{-1}\left[\left(e^{y}+\varphi_{1}-1\right)^{-2}+\left(e^{y}+\varphi_{2}-1\right)^{-2}\right]
$$

The elicited values of the hyper-parameters in the above prior predictive distribution are $\left(\varphi_{1}, \varphi_{2}\right)=(0.232768,0.322483)$. 


\section{FEROZE \& ASLAM}

\section{Results and Discussion}

A simulation study has been conducted to assess and compare the performance of Bayes estimators and to analyze the impact of sample size, mixing weight and magnitude of parametric values on the Bayes estimators. Samples of sizes $n=50$, 100, 200, 300, 400 and 500 have been generated by inverse transformation method from a two components mixture of the Gompertz distribution. The parametric values used are: $\left(\alpha_{1}, \alpha_{2}\right) \in\{(4,6),(8,12)\}$ and $p \in(0.45,0.60)$. Probabilistic mixing has been used to generate the mixture data. For each observation a random number $u$ has been generated from $U(0,1)$. If $u<p$ the observation has been randomly taken from first subpopulation and if $u>p$ then the observation has been taken from the second subpopulation. The observations above a fixed censoring time $T$ have been assumed to be right censored. Under each combination of parametric values, the choice of censoring time has been made so that the censoring rate in the respective sample is $15 \%$. As one sample cannot completely describe the behavior and properties of the Bayes estimators, the results have been replicated 1000 times and the average of results has been presented in the tables below (the amounts of posterior risks are presented in parenthesis). The abbreviations used in tables are; B.Es: Bayes estimates; P.Rs: posterior risks; $L L$ : lower limit and $U L$ : upper limit.

Table 1. B.Es and P.Rs under Uniform Prior

\begin{tabular}{|c|c|c|c|c|c|c|c|c|c|c|c|c|}
\hline & SELF & PLF & SELF & PLF & SELF & PLF & SELF & PLF & SELF & PLF & SELF & $P L F$ \\
\hline & \multicolumn{2}{|c|}{$\alpha_{1}=4, p=0.45$} & \multicolumn{2}{|c|}{$a_{1}=4, p=0.60$} & \multicolumn{2}{|c|}{$\alpha_{2}=6, p=0.45$} & \multicolumn{2}{|c|}{$a_{2}=6, p=0.60$} & \multicolumn{2}{|c|}{$p=0.45$} & \multicolumn{2}{|c|}{$p=0.60$} \\
\hline 50 & $\begin{array}{c}4.5131 \\
(0.2039)\end{array}$ & $\begin{array}{c}4.5363 \\
(0.0464)\end{array}$ & $\begin{array}{l}4.3991 \\
(0.1987)\end{array}$ & $\begin{array}{r}4.4217 \\
(0.0452)\end{array}$ & $\begin{array}{c}6.7921 \\
(0.3068)\end{array}$ & $\begin{array}{c}6.8271 \\
(0.0698)\end{array}$ & $\begin{array}{c}6.7307 \\
(0.3040)\end{array}$ & $\begin{array}{c}6.7653 \\
(0.0692)\end{array}$ & $\begin{array}{c}0.5190 \\
(0.0612)\end{array}$ & $\begin{array}{c}0.5217 \\
(0.0139)\end{array}$ & $\begin{array}{c}0.6709 \\
(0.0845)\end{array}$ & $\begin{array}{c}0.6743 \\
(0.0192)\end{array}$ \\
\hline 100 & $\begin{array}{r}4.4795 \\
(0.0989)\end{array}$ & $\begin{array}{r}4.4908 \\
(0.0227)\end{array}$ & $\begin{array}{r}4.3664 \\
(0.0964)\end{array}$ & $\begin{array}{c}4.3775 \\
(0.0221)\end{array}$ & $\begin{array}{c}6.7416 \\
(0.1488)\end{array}$ & $\begin{array}{c}6.7587 \\
(0.0342)\end{array}$ & $\begin{array}{c}6.6806 \\
(0.1475)\end{array}$ & $\begin{array}{c}6.6975 \\
(0.0339)\end{array}$ & $\begin{array}{c}0.5151 \\
(0.0297)\end{array}$ & $\begin{array}{c}0.5164 \\
(0.0068)\end{array}$ & $\begin{array}{c}0.6659 \\
(0.0410)\end{array}$ & $\begin{array}{c}0.6676 \\
(0.0094)\end{array}$ \\
\hline 200 & $\begin{array}{c}4.3936 \\
(0.0472)\end{array}$ & $\begin{array}{l}4.3991 \\
(0.0111)\end{array}$ & $\begin{array}{l}4.2827 \\
(0.0460)\end{array}$ & $\begin{array}{l}4.2881 \\
(0.0108)\end{array}$ & $\begin{array}{l}6.6124 \\
(0.0710)\end{array}$ & $\begin{array}{c}6.6207 \\
(0.0166)\end{array}$ & $\begin{array}{c}6.5525 \\
(0.0704)\end{array}$ & $\begin{array}{r}6.5608 \\
(0.0165)\end{array}$ & $\begin{array}{c}0.5053 \\
(0.0142)\end{array}$ & $\begin{array}{c}0.5059 \\
(0.0033)\end{array}$ & $\begin{array}{c}0.6531 \\
(0.0196)\end{array}$ & $\begin{array}{c}0.6539 \\
(0.0046)\end{array}$ \\
\hline 300 & $\begin{array}{c}4.2706 \\
(0.0297)\end{array}$ & $\begin{array}{c}4.2742 \\
(0.0072)\end{array}$ & $\begin{array}{l}4.1628 \\
(0.0289)\end{array}$ & $\begin{array}{c}4.1663 \\
(0.0070)\end{array}$ & $\begin{array}{c}6.4273 \\
(0.0446)\end{array}$ & $\begin{array}{l}6.4327 \\
(0.0108)\end{array}$ & $\begin{array}{c}6.3691 \\
(0.0442)\end{array}$ & $\begin{array}{r}6.3744 \\
(0.0107)\end{array}$ & $\begin{array}{c}0.4911 \\
(0.0089)\end{array}$ & $\begin{array}{c}0.4915 \\
(0.0021)\end{array}$ & $\begin{array}{c}0.6348 \\
(0.0123)\end{array}$ & $\begin{array}{c}0.6354 \\
(0.0030)\end{array}$ \\
\hline 400 & $\begin{array}{c}4.1707 \\
(0.0212)\end{array}$ & $\begin{array}{c}4.1734 \\
(0.0052)\end{array}$ & $\begin{array}{l}4.0655 \\
(0.0207)\end{array}$ & $\begin{array}{c}4.0680 \\
(0.0051)\end{array}$ & $\begin{array}{c}6.2770 \\
(0.0319)\end{array}$ & $\begin{array}{c}6.2809 \\
(0.0079)\end{array}$ & $\begin{array}{c}6.2202 \\
(0.0316)\end{array}$ & $\begin{array}{c}6.2241 \\
(0.0078)\end{array}$ & $\begin{array}{c}0.4796 \\
(0.0064)\end{array}$ & $\begin{array}{c}0.4799 \\
(0.0016)\end{array}$ & $\begin{array}{c}0.6200 \\
(0.0088)\end{array}$ & $\begin{array}{c}0.6204 \\
(0.0022)\end{array}$ \\
\hline 500 & $\begin{array}{c}4.1428 \\
(0.0167)\end{array}$ & $\begin{array}{c}4.1449 \\
(0.0042)\end{array}$ & $\begin{array}{c}4.0383 \\
(0.0163)\end{array}$ & $\begin{array}{c}4.0403 \\
(0.0040)\end{array}$ & $\begin{array}{c}6.2350 \\
(0.0252)\end{array}$ & $\begin{array}{c}6.2381 \\
(0.0063)\end{array}$ & $\begin{array}{c}6.1785 \\
(0.0249)\end{array}$ & $\begin{array}{c}6.1816 \\
(0.0062)\end{array}$ & $\begin{array}{c}0.4764 \\
(0.0050)\end{array}$ & $\begin{array}{c}0.4767 \\
(0.0012)\end{array}$ & $\begin{array}{c}0.6158 \\
(0.0069)\end{array}$ & $\begin{array}{c}0.6161 \\
(0.0017)\end{array}$ \\
\hline
\end{tabular}


ON PREDICTIONS FOR MIXTURE OF THE GOMPERTZ DISTRIBUTION

Table 2. B.Es and P.Rs under Uniform Prior

\begin{tabular}{|c|c|c|c|c|c|c|c|c|c|c|c|c|}
\hline & SELF & PLF & SELF & PLF & SELF & PLF & SELF & PLF & SELF & PLF & SELF & PLF \\
\hline & \multicolumn{2}{|c|}{$a_{1}=8, p=0.45$} & \multicolumn{2}{|c|}{$a_{1}=8, p=0.60$} & \multicolumn{2}{|c|}{$\alpha_{2}=12, p=0.45$} & \multicolumn{2}{|c|}{$\alpha_{2}=12, p=0.60$} & \multicolumn{2}{|c|}{$p=0.45$} & \multicolumn{2}{|c|}{$p=0.60$} \\
\hline 50 & $\begin{array}{c}8.8508 \\
(0.3998)\end{array}$ & $\begin{array}{c}8.8963 \\
(0.0910)\end{array}$ & $\begin{array}{c}8.8947 \\
(0.3563)\end{array}$ & $\begin{array}{l}8.9404 \\
(0.0811)\end{array}$ & $\begin{array}{l}13.3205 \\
(0.6017)\end{array}$ & $\begin{array}{l}13.3890 \\
(0.1370)\end{array}$ & $\begin{array}{l}13.4754 \\
(0.6769)\end{array}$ & $\begin{array}{l}13.5447 \\
(0.1541)\end{array}$ & $\begin{array}{c}0.5311 \\
(0.0760)\end{array}$ & $\begin{array}{c}0.5338 \\
(0.0173)\end{array}$ & $\begin{array}{c}0.7027 \\
(0.0994)\end{array}$ & $\begin{array}{c}0.7063 \\
(0.0226)\end{array}$ \\
\hline 100 & $\begin{array}{c}8.7850 \\
(0.1939)\end{array}$ & $\begin{array}{c}8.8072 \\
(0.0445)\end{array}$ & $\begin{array}{l}8.8284 \\
(0.1728)\end{array}$ & $\begin{array}{l}8.8508 \\
(0.0397)\end{array}$ & $\begin{array}{l}13.2214 \\
(0.2919)\end{array}$ & $\begin{array}{l}13.2549 \\
(0.0670)\end{array}$ & $\begin{array}{l}13.3751 \\
(0.3283)\end{array}$ & $\begin{array}{l}13.4090 \\
(0.0754)\end{array}$ & $\begin{array}{c}0.5271 \\
(0.0368)\end{array}$ & $\begin{array}{c}0.5284 \\
(0.0085)\end{array}$ & $\begin{array}{c}0.6974 \\
(0.0482)\end{array}$ & $\begin{array}{c}0.6992 \\
(0.0111)\end{array}$ \\
\hline 200 & $\begin{array}{c}8.6166 \\
(0.0926)\end{array}$ & $\begin{array}{c}8.6274 \\
(0.0217)\end{array}$ & $\begin{array}{c}8.6592 \\
(0.0825)\end{array}$ & $\begin{array}{l}8.6701 \\
(0.0193)\end{array}$ & $\begin{array}{l}12.9679 \\
(0.1393)\end{array}$ & $\begin{array}{l}12.9842 \\
(0.0326)\end{array}$ & $\begin{array}{l}13.1187 \\
(0.1567)\end{array}$ & $\begin{array}{l}13.1352 \\
(0.0367)\end{array}$ & $\begin{array}{c}0.5170 \\
(0.0176)\end{array}$ & $\begin{array}{c}0.5176 \\
(0.0041)\end{array}$ & $\begin{array}{c}0.6841 \\
(0.0230)\end{array}$ & $\begin{array}{c}0.6849 \\
(0.0054)\end{array}$ \\
\hline 300 & $\begin{array}{c}8.3754 \\
(0.0582)\end{array}$ & $\begin{array}{l}8.3824 \\
(0.0140)\end{array}$ & $\begin{array}{c}8.4168 \\
(0.0518)\end{array}$ & $\begin{array}{c}8.4239 \\
(0.0125)\end{array}$ & $\begin{array}{l}12.6050 \\
(0.0875)\end{array}$ & $\begin{array}{l}12.6155 \\
(0.0211)\end{array}$ & $\begin{array}{l}12.7515 \\
(0.0985)\end{array}$ & $\begin{array}{l}12.7622 \\
(0.0237)\end{array}$ & $\begin{array}{l}0.5025 \\
(0.0111)\end{array}$ & $\begin{array}{c}0.5029 \\
(0.0027)\end{array}$ & $\begin{array}{c}0.6649 \\
(0.0145)\end{array}$ & $\begin{array}{c}0.6655 \\
(0.0035)\end{array}$ \\
\hline 400 & $\begin{array}{c}8.1795 \\
(0.0416)\end{array}$ & $\begin{array}{c}8.1847 \\
(0.0103)\end{array}$ & $\begin{array}{l}8.2200 \\
(0.0370)\end{array}$ & $\begin{array}{l}8.2252 \\
(0.0091)\end{array}$ & $\begin{array}{l}12.3102 \\
(0.0625)\end{array}$ & $\begin{array}{l}12.3179 \\
(0.0154)\end{array}$ & $\begin{array}{l}12.4533 \\
(0.0704)\end{array}$ & $\begin{array}{l}12.4611 \\
(0.0174)\end{array}$ & $\begin{array}{c}0.4908 \\
(0.0079)\end{array}$ & $\begin{array}{c}0.4911 \\
(0.0019)\end{array}$ & $\begin{array}{c}0.6494 \\
(0.0103)\end{array}$ & $\begin{array}{r}0.6498 \\
(0.0026)\end{array}$ \\
\hline 500 & $\begin{array}{c}8.1248 \\
(0.0328)\end{array}$ & $\begin{array}{c}8.1289 \\
(0.0081)\end{array}$ & $\begin{array}{c}8.1650 \\
(0.0292)\end{array}$ & $\begin{array}{c}8.1691 \\
(0.0073)\end{array}$ & $\begin{array}{l}12.2278 \\
(0.0493)\end{array}$ & $\begin{array}{l}12.2339 \\
(0.0123)\end{array}$ & $\begin{array}{l}12.3700 \\
(0.0555)\end{array}$ & $\begin{array}{r}12.3762 \\
(0.0138)\end{array}$ & $\begin{array}{c}0.4875 \\
(0.0062)\end{array}$ & $\begin{array}{c}0.4877 \\
(0.0015)\end{array}$ & $\begin{array}{c}0.6450 \\
(0.0081)\end{array}$ & $\begin{array}{c}0.6454 \\
(0.0020)\end{array}$ \\
\hline
\end{tabular}

Table 3. B.Es and P.Rs under Jeffreys Prior

\begin{tabular}{|c|c|c|c|c|c|c|c|c|c|c|c|c|}
\hline & SELF & $P L F$ & SELF & PLF & SELF & PLF & SELF & PLF & SELF & PLF & SELF & PLF \\
\hline & \multicolumn{2}{|c|}{$\alpha_{1}=4, p=0.45$} & \multicolumn{2}{|c|}{$a_{1}=4, p=0.60$} & \multicolumn{2}{|c|}{$\alpha_{2}=6, p=0.45$} & \multicolumn{2}{|c|}{$a_{2}=6, p=0.60$} & \multicolumn{2}{|c|}{$p=0.45$} & \multicolumn{2}{|c|}{$p=0.60$} \\
\hline 50 & $\begin{array}{c}4.4679 \\
(0.2018)\end{array}$ & $\begin{array}{c}4.4909 \\
(0.0459)\end{array}$ & $\begin{array}{c}4.3551 \\
(0.1967)\end{array}$ & $\begin{array}{c}4.3775 \\
(0.0448)\end{array}$ & $\begin{array}{c}6.7242 \\
(0.3037)\end{array}$ & $\begin{array}{c}6.7588 \\
(0.0691)\end{array}$ & $\begin{array}{c}6.6634 \\
(0.3010)\end{array}$ & $\begin{array}{c}6.6976 \\
(0.0685)\end{array}$ & $\begin{array}{c}0.5138 \\
(0.0550)\end{array}$ & $\begin{array}{c}0.5165 \\
(0.0125)\end{array}$ & $\begin{array}{c}0.6642 \\
(0.0760)\end{array}$ & $\begin{array}{c}0.6676 \\
(0.0173)\end{array}$ \\
\hline 100 & $\begin{array}{c}4.4347 \\
(0.0979)\end{array}$ & $\begin{array}{c}4.4459 \\
(0.0225)\end{array}$ & $\begin{array}{c}4.3227 \\
(0.0954)\end{array}$ & $\begin{array}{c}4.3337 \\
(0.0219)\end{array}$ & $\begin{array}{c}6.6742 \\
(0.1473)\end{array}$ & $\begin{array}{c}6.6911 \\
(0.0338)\end{array}$ & $\begin{array}{c}6.6138 \\
(0.1460)\end{array}$ & $\begin{array}{c}6.6305 \\
(0.0335)\end{array}$ & $\begin{array}{c}0.5100 \\
(0.0267)\end{array}$ & $\begin{array}{c}0.5113 \\
(0.0061)\end{array}$ & $\begin{array}{c}0.6592 \\
(0.0369)\end{array}$ & $\begin{array}{c}0.6609 \\
(0.0085)\end{array}$ \\
\hline 200 & $\begin{array}{c}4.3497 \\
(0.0467)\end{array}$ & $\begin{array}{c}4.3551 \\
(0.0109)\end{array}$ & $\begin{array}{c}4.2399 \\
(0.0456)\end{array}$ & $\begin{array}{c}4.2452 \\
(0.0107)\end{array}$ & $\begin{array}{c}6.5462 \\
(0.0703)\end{array}$ & $\begin{array}{c}6.5545 \\
(0.0165)\end{array}$ & $\begin{array}{c}6.4870 \\
(0.0697)\end{array}$ & $\begin{array}{c}6.4951 \\
(0.0163)\end{array}$ & $\begin{array}{c}0.5002 \\
(0.0127)\end{array}$ & $\begin{array}{c}0.5008 \\
(0.0030)\end{array}$ & $\begin{array}{c}0.6466 \\
(0.0176)\end{array}$ & $\begin{array}{c}0.6474 \\
(0.0041)\end{array}$ \\
\hline 300 & $\begin{array}{c}4.2279 \\
(0.0294)\end{array}$ & $\begin{array}{c}4.2314 \\
(0.0071)\end{array}$ & $\begin{array}{c}4.1212 \\
(0.0286)\end{array}$ & $\begin{array}{c}4.1246 \\
(0.0069)\end{array}$ & $\begin{array}{c}6.3630 \\
(0.0442)\end{array}$ & $\begin{array}{c}6.3683 \\
(0.0107)\end{array}$ & $\begin{array}{c}6.3054 \\
(0.0438)\end{array}$ & $\begin{array}{c}6.3107 \\
(0.0106)\end{array}$ & $\begin{array}{c}0.4862 \\
(0.0080)\end{array}$ & $\begin{array}{c}0.4866 \\
(0.0019)\end{array}$ & $\begin{array}{c}0.6285 \\
(0.0111)\end{array}$ & $\begin{array}{c}0.6290 \\
(0.0027)\end{array}$ \\
\hline 400 & $\begin{array}{c}4.1290 \\
(0.0210)\end{array}$ & $\begin{array}{l}4.1316 \\
(0.0052)\end{array}$ & $\begin{array}{c}4.0248 \\
(0.0204)\end{array}$ & $\begin{array}{c}4.0273 \\
(0.0050)\end{array}$ & $\begin{array}{c}6.2142 \\
(0.0316)\end{array}$ & $\begin{array}{c}6.2181 \\
(0.0078)\end{array}$ & $\begin{array}{c}6.1580 \\
(0.0313)\end{array}$ & $\begin{array}{c}6.1618 \\
(0.0077)\end{array}$ & $\begin{array}{c}0.4748 \\
(0.0057)\end{array}$ & $\begin{array}{c}0.4751 \\
(0.0014)\end{array}$ & $\begin{array}{c}0.6138 \\
(0.0079)\end{array}$ & $\begin{array}{c}0.6142 \\
(0.0020)\end{array}$ \\
\hline 500 & $\begin{array}{l}4.1014 \\
(0.0165)\end{array}$ & $\begin{array}{l}4.1035 \\
(0.0041)\end{array}$ & $\begin{array}{c}3.9979 \\
(0.0161)\end{array}$ & $\begin{array}{r}3.9999 \\
(0.0040)\end{array}$ & $\begin{array}{c}6.1726 \\
(0.0249)\end{array}$ & $\begin{array}{c}6.1757 \\
(0.0062)\end{array}$ & $\begin{array}{c}6.1167 \\
(0.0247)\end{array}$ & $\begin{array}{c}6.1198 \\
(0.0061)\end{array}$ & $\begin{array}{c}0.4717 \\
(0.0045)\end{array}$ & $\begin{array}{c}0.4719 \\
(0.0011)\end{array}$ & $\begin{array}{l}0.6097 \\
(0.0062)\end{array}$ & $\begin{array}{l}0.6100 \\
(0.0015)\end{array}$ \\
\hline
\end{tabular}




\section{FEROZE \& ASLAM}

Table 4. B.Es and P.Rs under Jeffreys Prior

\begin{tabular}{|c|c|c|c|c|c|c|c|c|c|c|c|c|}
\hline \multirow{2}{*}{$n$} & SELF & PLF & SELF & PLF & SELF & PLF & SELF & PLF & SELF & PLF & SELF & PLF \\
\hline & \multicolumn{2}{|c|}{$a_{1}=8, p=0.45$} & \multicolumn{2}{|c|}{$a_{1}=8, p=0.60$} & \multicolumn{2}{|c|}{$\alpha_{2}=12, p=0.45$} & \multicolumn{2}{|c|}{$\alpha_{2}=12, p=0.60$} & \multicolumn{2}{|c|}{$p=0.45$} & \multicolumn{2}{|c|}{$p=0.60$} \\
\hline 50 & $\begin{array}{c}8.7623 \\
(0.3958)\end{array}$ & $\begin{array}{l}8.8074 \\
(0.0901)\end{array}$ & $\begin{array}{c}8.8057 \\
(0.3527)\end{array}$ & $\begin{array}{l}8.8510 \\
(0.0803)\end{array}$ & $\begin{array}{l}13.1873 \\
(0.5957)\end{array}$ & $\begin{array}{l}13.2551 \\
(0.1356)\end{array}$ & $\begin{array}{l}13.3406 \\
(0.6701)\end{array}$ & $\begin{array}{l}13.4092 \\
(0.1525)\end{array}$ & $\begin{array}{c}0.5257 \\
(0.0684)\end{array}$ & $\begin{array}{c}0.5284 \\
(0.0156)\end{array}$ & $\begin{array}{c}0.6957 \\
(0.0895)\end{array}$ & $\begin{array}{c}0.6992 \\
(0.0204)\end{array}$ \\
\hline 100 & $\begin{array}{c}8.6971 \\
(0.1920)\end{array}$ & $\begin{array}{c}8.7191 \\
(0.0441)\end{array}$ & $\begin{array}{l}8.7402 \\
(0.1711)\end{array}$ & $\begin{array}{c}8.7623 \\
(0.0393)\end{array}$ & $\begin{array}{l}13.0891 \\
(0.2890)\end{array}$ & $\begin{array}{l}13.1223 \\
(0.0664)\end{array}$ & $\begin{array}{r}13.2413 \\
(0.3251)\end{array}$ & $\begin{array}{l}13.2749 \\
(0.0747)\end{array}$ & $\begin{array}{c}0.5218 \\
(0.0332)\end{array}$ & $\begin{array}{c}0.5231 \\
(0.0076)\end{array}$ & $\begin{array}{c}0.6905 \\
(0.0434)\end{array}$ & $\begin{array}{c}0.6922 \\
(0.0100)\end{array}$ \\
\hline 200 & $\begin{array}{l}8.5304 \\
(0.0917)\end{array}$ & $\begin{array}{c}8.5411 \\
(0.0215)\end{array}$ & $\begin{array}{c}8.5726 \\
(0.0817)\end{array}$ & $\begin{array}{l}8.5834 \\
(0.0191)\end{array}$ & $\begin{array}{l}12.8382 \\
(0.1379)\end{array}$ & $\begin{array}{l}12.8544 \\
(0.0323)\end{array}$ & $\begin{array}{r}12.9875 \\
(0.1552)\end{array}$ & $\begin{array}{l}13.0039 \\
(0.0364)\end{array}$ & $\begin{array}{c}0.5118 \\
(0.0158)\end{array}$ & $\begin{array}{c}0.5125 \\
(0.0037)\end{array}$ & $\begin{array}{c}0.6772 \\
(0.0207)\end{array}$ & $\begin{array}{c}0.6781 \\
(0.0049)\end{array}$ \\
\hline 300 & $\begin{array}{c}8.2916 \\
(0.0576)\end{array}$ & $\begin{array}{c}8.2986 \\
(0.0139)\end{array}$ & $\begin{array}{c}8.3327 \\
(0.0513)\end{array}$ & $\begin{array}{c}8.3397 \\
(0.0124)\end{array}$ & $\begin{array}{l}12.4789 \\
(0.0867)\end{array}$ & $\begin{array}{l}12.4893 \\
(0.0209)\end{array}$ & $\begin{array}{r}12.6240 \\
(0.0975)\end{array}$ & $\begin{array}{l}12.6346 \\
(0.0235)\end{array}$ & $\begin{array}{c}0.4975 \\
(0.0099)\end{array}$ & $\begin{array}{c}0.4979 \\
(0.0024)\end{array}$ & $\begin{array}{c}0.6583 \\
(0.0130)\end{array}$ & $\begin{array}{c}0.6588 \\
(0.0031)\end{array}$ \\
\hline 400 & $\begin{array}{l}8.0977 \\
(0.0411)\end{array}$ & $\begin{array}{l}8.1028 \\
(0.0102)\end{array}$ & $\begin{array}{c}8.1378 \\
(0.0367)\end{array}$ & $\begin{array}{l}8.1429 \\
(0.0091)\end{array}$ & $\begin{array}{l}12.1871 \\
(0.0619)\end{array}$ & $\begin{array}{l}12.1947 \\
(0.0153)\end{array}$ & $\begin{array}{l}12.3288 \\
(0.0697)\end{array}$ & $\begin{array}{l}12.3365 \\
(0.0172)\end{array}$ & $\begin{array}{c}0.4859 \\
(0.0071)\end{array}$ & $\begin{array}{c}0.4862 \\
(0.0018)\end{array}$ & $\begin{array}{c}0.6429 \\
(0.0093)\end{array}$ & $\begin{array}{c}0.6433 \\
(0.0023)\end{array}$ \\
\hline 500 & $\begin{array}{c}8.0435 \\
(0.0324)\end{array}$ & $\begin{array}{c}8.0476 \\
(0.0081)\end{array}$ & $\begin{array}{c}8.0834 \\
(0.0289)\end{array}$ & $\begin{array}{c}8.0874 \\
(0.0072)\end{array}$ & $\begin{array}{l}12.1055 \\
(0.0488)\end{array}$ & $\begin{array}{l}12.1116 \\
(0.0121)\end{array}$ & $\begin{array}{l}12.2463 \\
(0.0549)\end{array}$ & $\begin{array}{l}12.2524 \\
(0.0137)\end{array}$ & $\begin{array}{c}0.4826 \\
(0.0056)\end{array}$ & $\begin{array}{c}0.4829 \\
(0.0014)\end{array}$ & $\begin{array}{c}0.6386 \\
(0.0073)\end{array}$ & $\begin{array}{c}0.6389 \\
(0.0018)\end{array}$ \\
\hline
\end{tabular}

Table 5. B.Es and P.Rs under Gamma Prior

\begin{tabular}{|c|c|c|c|c|c|c|c|c|c|c|c|c|}
\hline \multirow{2}{*}{$n$} & SELF & PLF & SELF & PLF & SELF & PLF & SELF & PLF & SELF & PLF & SELF & PLF \\
\hline & \multicolumn{2}{|c|}{$a_{1}=4, p=0.45$} & \multicolumn{2}{|c|}{$a_{1}=4, p=0.60$} & \multicolumn{2}{|c|}{$\alpha_{2}=6, p=0.45$} & \multicolumn{2}{|c|}{$\alpha_{2}=6, p=0.60$} & \multicolumn{2}{|c|}{$p=0.45$} & \multicolumn{2}{|c|}{$p=0.60$} \\
\hline 50 & $\begin{array}{c}4.3777 \\
(0.1835)\end{array}$ & $\begin{array}{l}4.4002 \\
(0.0418)\end{array}$ & $\begin{array}{c}4.2672 \\
(0.1788)\end{array}$ & $\begin{array}{l}4.2891 \\
(0.0407)\end{array}$ & $\begin{array}{c}6.5884 \\
(0.2761)\end{array}$ & $\begin{array}{c}6.6222 \\
(0.0629)\end{array}$ & $\begin{array}{c}6.5287 \\
(0.2736)\end{array}$ & $\begin{array}{c}6.5623 \\
(0.0623)\end{array}$ & $\begin{array}{c}0.5034 \\
(0.0428)\end{array}$ & $\begin{array}{c}0.5060 \\
(0.0097)\end{array}$ & $\begin{array}{c}0.6507 \\
(0.0591)\end{array}$ & $\begin{array}{l}0.6541 \\
(0.0135)\end{array}$ \\
\hline 100 & $\begin{array}{c}4.3451 \\
(0.0890)\end{array}$ & $\begin{array}{c}4.3561 \\
(0.0204)\end{array}$ & $\begin{array}{c}4.2354 \\
(0.0868)\end{array}$ & $\begin{array}{c}4.2461 \\
(0.0199)\end{array}$ & $\begin{array}{c}6.5393 \\
(0.1339)\end{array}$ & $\begin{array}{c}6.5559 \\
(0.0308)\end{array}$ & $\begin{array}{c}6.4801 \\
(0.1327)\end{array}$ & $\begin{array}{c}6.4966 \\
(0.0305)\end{array}$ & $\begin{array}{c}0.4997 \\
(0.0208)\end{array}$ & $\begin{array}{c}0.5009 \\
(0.0048)\end{array}$ & $\begin{array}{c}0.6459 \\
(0.0287)\end{array}$ & $\begin{array}{c}0.6475 \\
(0.0066)\end{array}$ \\
\hline 200 & $\begin{array}{c}4.2618 \\
(0.0425)\end{array}$ & $\begin{array}{l}4.2671 \\
(0.0100)\end{array}$ & $\begin{array}{l}4.1542 \\
(0.0414)\end{array}$ & $\begin{array}{l}4.1594 \\
(0.0097)\end{array}$ & $\begin{array}{c}6.4140 \\
(0.0639)\end{array}$ & $\begin{array}{c}6.4221 \\
(0.0150)\end{array}$ & $\begin{array}{c}6.3559 \\
(0.0634)\end{array}$ & $\begin{array}{c}6.3639 \\
(0.0148)\end{array}$ & $\begin{array}{l}0.4901 \\
(0.0099)\end{array}$ & $\begin{array}{c}0.4907 \\
(0.0023)\end{array}$ & $\begin{array}{c}0.6335 \\
(0.0137)\end{array}$ & $\begin{array}{l}0.6343 \\
(0.0032)\end{array}$ \\
\hline 300 & $\begin{array}{l}4.1425 \\
(0.0267)\end{array}$ & $\begin{array}{l}4.1460 \\
(0.0064)\end{array}$ & $\begin{array}{l}4.0379 \\
(0.0260)\end{array}$ & $\begin{array}{c}4.0413 \\
(0.0063)\end{array}$ & $\begin{array}{c}6.2345 \\
(0.0402)\end{array}$ & $\begin{array}{c}6.2397 \\
(0.0097)\end{array}$ & $\begin{array}{c}6.1780 \\
(0.0398)\end{array}$ & $\begin{array}{c}6.1832 \\
(0.0096)\end{array}$ & $\begin{array}{l}0.4764 \\
(0.0062)\end{array}$ & $\begin{array}{c}0.4768 \\
(0.0015)\end{array}$ & $\begin{array}{c}0.6158 \\
(0.0086)\end{array}$ & $\begin{array}{c}0.6163 \\
(0.0021)\end{array}$ \\
\hline 400 & $\begin{array}{c}4.0456 \\
(0.0191)\end{array}$ & $\begin{array}{l}4.0482 \\
(0.0047)\end{array}$ & $\begin{array}{l}3.9435 \\
(0.0186)\end{array}$ & $\begin{array}{c}3.9460 \\
(0.0046)\end{array}$ & $\begin{array}{c}6.0887 \\
(0.0287)\end{array}$ & $\begin{array}{c}6.0925 \\
(0.0071)\end{array}$ & $\begin{array}{c}6.0336 \\
(0.0284)\end{array}$ & $\begin{array}{c}6.0373 \\
(0.0070)\end{array}$ & $\begin{array}{c}0.4652 \\
(0.0044)\end{array}$ & $\begin{array}{c}0.4655 \\
(0.0011)\end{array}$ & $\begin{array}{c}0.6014 \\
(0.0061)\end{array}$ & $\begin{array}{c}0.6018 \\
(0.0015)\end{array}$ \\
\hline 500 & $\begin{array}{r}4.0185 \\
(0.0150)\end{array}$ & $\begin{array}{l}4.0206 \\
(0.0037)\end{array}$ & $\begin{array}{c}3.9171 \\
(0.0147)\end{array}$ & $\begin{array}{c}3.9191 \\
(0.0036)\end{array}$ & $\begin{array}{c}6.0479 \\
(0.0226)\end{array}$ & $\begin{array}{c}6.0509 \\
(0.0056)\end{array}$ & $\begin{array}{c}5.9932 \\
(0.0224)\end{array}$ & $\begin{array}{r}5.9962 \\
(0.0056)\end{array}$ & $\begin{array}{l}0.4621 \\
(0.0035)\end{array}$ & $\begin{array}{c}0.4624 \\
(0.0009)\end{array}$ & $\begin{array}{c}0.5974 \\
(0.0048)\end{array}$ & $\begin{array}{c}0.5977 \\
(0.0012)\end{array}$ \\
\hline
\end{tabular}


ON PREDICTIONS FOR MIXTURE OF THE GOMPERTZ DISTRIBUTION

Table 6. B.Es and P.Rs under Gamma Prior

\begin{tabular}{|c|c|c|c|c|c|c|c|c|c|c|c|c|}
\hline & SELF & PLF & SELF & PLF & SELF & PLF & SELF & PLF & SELF & PLF & SELF & PLF \\
\hline & \multicolumn{2}{|c|}{$a_{1}=8, p=0.45$} & \multicolumn{2}{|c|}{$\alpha_{1}=8, p=0.60$} & \multicolumn{2}{|c|}{$a_{2}=12, p=0.45$} & \multicolumn{2}{|c|}{$\alpha_{2}=12, p=0.60$} & \multicolumn{2}{|c|}{$p=0.45$} & \multicolumn{2}{|c|}{$p=0.60$} \\
\hline 50 & $\begin{array}{c}8.5853 \\
(0.3598)\end{array}$ & $\begin{array}{l}8.6295 \\
(0.0819)\end{array}$ & $\begin{array}{c}8.6278 \\
(0.3206)\end{array}$ & $\begin{array}{l}8.6722 \\
(0.0730)\end{array}$ & $\begin{array}{l}12.9209 \\
(0.5415)\end{array}$ & $\begin{array}{l}12.9873 \\
(0.1233)\end{array}$ & $\begin{array}{l}13.0711 \\
(0.6092)\end{array}$ & $\begin{array}{l}13.1383 \\
(0.1387)\end{array}$ & $\begin{array}{c}0.5151 \\
(0.0532)\end{array}$ & $\begin{array}{c}0.5178 \\
(0.0121)\end{array}$ & $\begin{array}{c}0.6816 \\
(0.0696)\end{array}$ & $\begin{array}{c}0.6851 \\
(0.0158)\end{array}$ \\
\hline 100 & $\begin{array}{c}8.5214 \\
(0.1745)\end{array}$ & $\begin{array}{c}8.5430 \\
(0.0401)\end{array}$ & $\begin{array}{c}8.5636 \\
(0.1555)\end{array}$ & $\begin{array}{l}8.5853 \\
(0.0357)\end{array}$ & $\begin{array}{l}12.8247 \\
(0.2627)\end{array}$ & $\begin{array}{l}12.8572 \\
(0.0603)\end{array}$ & $\begin{array}{l}12.9738 \\
(0.2955)\end{array}$ & $\begin{array}{l}13.0067 \\
(0.0679)\end{array}$ & $\begin{array}{c}0.5113 \\
(0.0258)\end{array}$ & $\begin{array}{c}0.5126 \\
(0.0059)\end{array}$ & $\begin{array}{c}0.6765 \\
(0.0338)\end{array}$ & $\begin{array}{c}0.6782 \\
(0.0078)\end{array}$ \\
\hline 200 & $\begin{array}{c}8.3581 \\
(0.0833)\end{array}$ & $\begin{array}{c}8.3686 \\
(0.0195)\end{array}$ & $\begin{array}{c}8.3994 \\
(0.0742)\end{array}$ & $\begin{array}{c}8.4100 \\
(0.0174)\end{array}$ & $\begin{array}{l}12.5789 \\
(0.1254)\end{array}$ & $\begin{array}{l}12.5947 \\
(0.0294)\end{array}$ & $\begin{array}{l}12.7251 \\
(0.1411)\end{array}$ & $\begin{array}{l}12.7412 \\
(0.0331)\end{array}$ & $\begin{array}{c}0.5015 \\
(0.0123)\end{array}$ & $\begin{array}{c}0.5021 \\
(0.0029)\end{array}$ & $\begin{array}{c}0.6636 \\
(0.0161)\end{array}$ & $\begin{array}{c}0.6644 \\
(0.0038)\end{array}$ \\
\hline 300 & $\begin{array}{c}8.1241 \\
(0.0524)\end{array}$ & $\begin{array}{r}8.1309 \\
(0.0126)\end{array}$ & $\begin{array}{r}8.1643 \\
(0.0466)\end{array}$ & $\begin{array}{c}8.1712 \\
(0.0112)\end{array}$ & $\begin{array}{l}12.2268 \\
(0.0788)\end{array}$ & $\begin{array}{l}12.2370 \\
(0.0190)\end{array}$ & $\begin{array}{l}12.3690 \\
(0.0886)\end{array}$ & $\begin{array}{l}12.3793 \\
(0.0214)\end{array}$ & $\begin{array}{r}0.4874 \\
(0.0077)\end{array}$ & $\begin{array}{c}0.4879 \\
(0.0019)\end{array}$ & $\begin{array}{c}0.6450 \\
(0.0101)\end{array}$ & $\begin{array}{c}0.6455 \\
(0.0024)\end{array}$ \\
\hline 400 & $\begin{array}{c}7.9341 \\
(0.0374)\end{array}$ & $\begin{array}{c}7.9391 \\
(0.0092)\end{array}$ & $\begin{array}{c}7.9734 \\
(0.0333)\end{array}$ & $\begin{array}{l}7.9784 \\
(0.0082)\end{array}$ & $\begin{array}{l}11.9409 \\
(0.0563)\end{array}$ & $\begin{array}{l}11.9484 \\
(0.0139)\end{array}$ & $\begin{array}{l}12.0797 \\
(0.0633)\end{array}$ & $\begin{array}{l}12.0873 \\
(0.0156)\end{array}$ & $\begin{array}{l}0.4760 \\
(0.0055)\end{array}$ & $\begin{array}{c}0.4763 \\
(0.0014)\end{array}$ & $\begin{array}{c}0.6299 \\
(0.0072)\end{array}$ & $\begin{array}{r}0.6303 \\
(0.0018)\end{array}$ \\
\hline 500 & $\begin{array}{c}7.8810 \\
(0.0295)\end{array}$ & $\begin{array}{c}7.8850 \\
(0.0073)\end{array}$ & $\begin{array}{c}7.9201 \\
(0.0263)\end{array}$ & $\begin{array}{c}7.9240 \\
(0.0065)\end{array}$ & $\begin{array}{l}11.8610 \\
(0.0444)\end{array}$ & $\begin{array}{l}11.8669 \\
(0.0110)\end{array}$ & $\begin{array}{l}11.9989 \\
(0.0499)\end{array}$ & $\begin{array}{l}12.0049 \\
(0.0124)\end{array}$ & $\begin{array}{c}0.4729 \\
(0.0044)\end{array}$ & $\begin{array}{c}0.4731 \\
(0.0011)\end{array}$ & $\begin{array}{c}0.6257 \\
(0.0057)\end{array}$ & $\begin{array}{c}0.6260 \\
(0.0014)\end{array}$ \\
\hline
\end{tabular}

Table 7. B.Es and P.Rs under Chi Square Prior

\begin{tabular}{|c|c|c|c|c|c|c|c|c|c|c|c|c|}
\hline & SELF & PLF & SELF & PLF & SELF & PLF & SELF & PLF & SELF & PLF & SELF & PLF \\
\hline & \multicolumn{2}{|c|}{$a_{1}=4, p=0.45$} & \multicolumn{2}{|c|}{$a_{1}=4, p=0.60$} & \multicolumn{2}{|c|}{$\alpha_{2}=6, p=0.45$} & \multicolumn{2}{|c|}{$\alpha_{2}=6, p=0.60$} & \multicolumn{2}{|c|}{$p=0.45$} & \multicolumn{2}{|c|}{$p=0.60$} \\
\hline 50 & $\begin{array}{c}4.4228 \\
(0.1876)\end{array}$ & $\begin{array}{c}4.4455 \\
(0.0427)\end{array}$ & $\begin{array}{c}4.3111 \\
(0.1828)\end{array}$ & $\begin{array}{c}4.3333 \\
(0.0416)\end{array}$ & $\begin{array}{c}6.6563 \\
(0.2823)\end{array}$ & $\begin{array}{c}6.6905 \\
(0.0643)\end{array}$ & $\begin{array}{c}6.5961 \\
(0.2797)\end{array}$ & $\begin{array}{c}6.6300 \\
(0.0637)\end{array}$ & $\begin{array}{c}0.5086 \\
(0.0489)\end{array}$ & $\begin{array}{c}0.5112 \\
(0.0111)\end{array}$ & $\begin{array}{c}0.6574 \\
(0.0676)\end{array}$ & $\begin{array}{c}0.6608 \\
(0.0154)\end{array}$ \\
\hline 100 & $\begin{array}{c}4.3899 \\
(0.0910)\end{array}$ & $\begin{array}{c}4.4010 \\
(0.0209)\end{array}$ & $\begin{array}{c}4.2791 \\
(0.0887)\end{array}$ & $\begin{array}{c}4.2899 \\
(0.0204)\end{array}$ & $\begin{array}{c}6.6067 \\
(0.1369)\end{array}$ & $\begin{array}{c}6.6235 \\
(0.0314)\end{array}$ & $\begin{array}{r}6.5470 \\
(0.1357)\end{array}$ & $\begin{array}{c}6.5635 \\
(0.0312)\end{array}$ & $\begin{array}{c}0.5048 \\
(0.0237)\end{array}$ & $\begin{array}{c}0.5061 \\
(0.0055)\end{array}$ & $\begin{array}{c}0.6526 \\
(0.0328)\end{array}$ & $\begin{array}{c}0.6542 \\
(0.0075)\end{array}$ \\
\hline 200 & $\begin{array}{c}4.3057 \\
(0.0434)\end{array}$ & $\begin{array}{c}4.3111 \\
(0.0102)\end{array}$ & $\begin{array}{c}4.1970 \\
(0.0423)\end{array}$ & $\begin{array}{c}4.2023 \\
(0.0099)\end{array}$ & $\begin{array}{c}6.4801 \\
(0.0654)\end{array}$ & $\begin{array}{c}6.4883 \\
(0.0153)\end{array}$ & $\begin{array}{c}6.4215 \\
(0.0648)\end{array}$ & $\begin{array}{c}6.4295 \\
(0.0152)\end{array}$ & $\begin{array}{c}0.4952 \\
(0.0113)\end{array}$ & $\begin{array}{c}0.4958 \\
(0.0027)\end{array}$ & $\begin{array}{c}0.6400 \\
(0.0156)\end{array}$ & $\begin{array}{c}0.6409 \\
(0.0037)\end{array}$ \\
\hline 300 & $\begin{array}{c}4.1852 \\
(0.0273)\end{array}$ & $\begin{array}{l}4.1887 \\
(0.0066)\end{array}$ & $\begin{array}{c}4.0796 \\
(0.0266)\end{array}$ & $\begin{array}{c}4.0830 \\
(0.0064)\end{array}$ & $\begin{array}{c}6.2987 \\
(0.0411)\end{array}$ & $\begin{array}{c}6.3040 \\
(0.0099)\end{array}$ & $\begin{array}{c}6.2417 \\
(0.0407)\end{array}$ & $\begin{array}{c}6.2469 \\
(0.0098)\end{array}$ & $\begin{array}{c}0.4813 \\
(0.0071)\end{array}$ & $\begin{array}{c}0.4817 \\
(0.0017)\end{array}$ & $\begin{array}{c}0.6221 \\
(0.0098)\end{array}$ & $\begin{array}{c}0.6227 \\
(0.0024)\end{array}$ \\
\hline 400 & $\begin{array}{c}4.0873 \\
(0.0195)\end{array}$ & $\begin{array}{l}4.0899 \\
(0.0048)\end{array}$ & $\begin{array}{l}3.9842 \\
(0.0190)\end{array}$ & $\begin{array}{l}3.9867 \\
(0.0047)\end{array}$ & $\begin{array}{c}6.1514 \\
(0.0293)\end{array}$ & $\begin{array}{c}6.1553 \\
(0.0072)\end{array}$ & $\begin{array}{r}6.0958 \\
(0.0291)\end{array}$ & $\begin{array}{c}6.0996 \\
(0.0072)\end{array}$ & $\begin{array}{c}0.4700 \\
(0.0051)\end{array}$ & $\begin{array}{c}0.4703 \\
(0.0013)\end{array}$ & $\begin{array}{c}0.6076 \\
(0.0070)\end{array}$ & $\begin{array}{c}0.6080 \\
(0.0017)\end{array}$ \\
\hline 500 & $\begin{array}{c}4.0600 \\
(0.0154)\end{array}$ & $\begin{array}{c}4.0620 \\
(0.0038)\end{array}$ & $\begin{array}{c}3.9575 \\
(0.0150)\end{array}$ & $\begin{array}{c}3.9595 \\
(0.0037)\end{array}$ & $\begin{array}{c}6.1103 \\
(0.0231)\end{array}$ & $\begin{array}{c}6.1133 \\
(0.0058)\end{array}$ & $\begin{array}{r}6.0550 \\
(0.0229)\end{array}$ & $\begin{array}{c}6.0580 \\
(0.0057)\end{array}$ & $\begin{array}{c}0.4669 \\
(0.0040)\end{array}$ & $\begin{array}{l}0.4671 \\
(0.0010)\end{array}$ & $\begin{array}{r}0.6035 \\
(0.0055)\end{array}$ & $\begin{array}{c}0.6038 \\
(0.0014)\end{array}$ \\
\hline
\end{tabular}




\section{FEROZE \& ASLAM}

Table 8. B.Es and P.Rs under Chi Square Prior

\begin{tabular}{|c|c|c|c|c|c|c|c|c|c|c|c|c|}
\hline \multirow{2}{*}{$n$} & SELF & PLF & SELF & PLF & SELF & PLF & SELF & PLF & SELF & PLF & SELF & PLF \\
\hline & \multicolumn{2}{|c|}{$a_{1}=8, p=0.45$} & \multicolumn{2}{|c|}{$a_{1}=8, p=0.60$} & \multicolumn{2}{|c|}{$\alpha_{2}=12, p=0.45$} & \multicolumn{2}{|c|}{$\alpha_{2}=12, p=0.60$} & \multicolumn{2}{|c|}{$p=0.45$} & \multicolumn{2}{|c|}{$p=0.60$} \\
\hline 50 & $\begin{array}{c}8.6738 \\
(0.3678)\end{array}$ & $\begin{array}{c}8.7184 \\
(0.0837)\end{array}$ & $\begin{array}{c}8.7168 \\
(0.3278)\end{array}$ & $\begin{array}{c}8.7616 \\
(0.0746)\end{array}$ & $\begin{array}{l}13.0541 \\
(0.5536)\end{array}$ & $\begin{array}{l}13.1212 \\
(0.1260)\end{array}$ & $\begin{array}{r}13.2059 \\
(0.6227)\end{array}$ & $\begin{array}{l}13.2738 \\
(0.1418)\end{array}$ & $\begin{array}{l}0.5204 \\
(0.0608)\end{array}$ & $\begin{array}{c}0.5231 \\
(0.0138)\end{array}$ & $\begin{array}{c}0.6886 \\
(0.0795)\end{array}$ & $\begin{array}{c}0.6922 \\
(0.0181)\end{array}$ \\
\hline 100 & $\begin{array}{c}8.6093 \\
(0.1784)\end{array}$ & $\begin{array}{c}8.6311 \\
(0.0410)\end{array}$ & $\begin{array}{l}8.6519 \\
(0.1590)\end{array}$ & $\begin{array}{c}8.6738 \\
(0.0365)\end{array}$ & $\begin{array}{l}12.9569 \\
(0.2685)\end{array}$ & $\begin{array}{l}12.9898 \\
(0.0617)\end{array}$ & $\begin{array}{r}13.1076 \\
(0.3021)\end{array}$ & $\begin{array}{l}13.1408 \\
(0.0694)\end{array}$ & $\begin{array}{c}0.5166 \\
(0.0295)\end{array}$ & $\begin{array}{c}0.5179 \\
(0.0068)\end{array}$ & $\begin{array}{c}0.6835 \\
(0.0386)\end{array}$ & $\begin{array}{c}0.6852 \\
(0.0089)\end{array}$ \\
\hline 200 & $\begin{array}{c}8.4442 \\
(0.0852)\end{array}$ & $\begin{array}{c}8.4549 \\
(0.0200)\end{array}$ & $\begin{array}{c}8.4860 \\
(0.0759)\end{array}$ & $\begin{array}{c}8.4967 \\
(0.0178)\end{array}$ & $\begin{array}{l}12.7086 \\
(0.1282)\end{array}$ & $\begin{array}{l}12.7246 \\
(0.0300)\end{array}$ & $\begin{array}{r}12.8563 \\
(0.1442)\end{array}$ & $\begin{array}{l}12.8725 \\
(0.0338)\end{array}$ & $\begin{array}{c}0.5067 \\
(0.0141)\end{array}$ & $\begin{array}{c}0.5073 \\
(0.0033)\end{array}$ & $\begin{array}{c}0.6704 \\
(0.0184)\end{array}$ & $\begin{array}{c}0.6712 \\
(0.0043)\end{array}$ \\
\hline 300 & $\begin{array}{l}8.2079 \\
(0.0535)\end{array}$ & $\begin{array}{c}8.2147 \\
(0.0129)\end{array}$ & $\begin{array}{c}8.2485 \\
(0.0477)\end{array}$ & $\begin{array}{l}8.2554 \\
(0.0115)\end{array}$ & $\begin{array}{l}12.3529 \\
(0.0805)\end{array}$ & $\begin{array}{l}12.3632 \\
(0.0194)\end{array}$ & $\begin{array}{r}12.4965 \\
(0.0906)\end{array}$ & $\begin{array}{l}12.5070 \\
(0.0218)\end{array}$ & $\begin{array}{c}0.4925 \\
(0.0088)\end{array}$ & $\begin{array}{c}0.4929 \\
(0.0021)\end{array}$ & $\begin{array}{c}0.6516 \\
(0.0116)\end{array}$ & $\begin{array}{c}0.6522 \\
(0.0028)\end{array}$ \\
\hline 400 & $\begin{array}{l}8.0159 \\
(0.0382)\end{array}$ & $\begin{array}{l}8.0210 \\
(0.0094)\end{array}$ & $\begin{array}{l}8.0556 \\
(0.0341)\end{array}$ & $\begin{array}{c}8.0607 \\
(0.0084)\end{array}$ & $\begin{array}{l}12.0640 \\
(0.0575)\end{array}$ & $\begin{array}{l}12.0715 \\
(0.0142)\end{array}$ & $\begin{array}{l}12.2043 \\
(0.0647)\end{array}$ & $\begin{array}{l}12.2119 \\
(0.0160)\end{array}$ & $\begin{array}{c}0.4810 \\
(0.0063)\end{array}$ & $\begin{array}{c}0.4813 \\
(0.0016)\end{array}$ & $\begin{array}{c}0.6364 \\
(0.0083)\end{array}$ & $\begin{array}{c}0.6368 \\
(0.0020)\end{array}$ \\
\hline 500 & $\begin{array}{c}7.9623 \\
(0.0302)\end{array}$ & $\begin{array}{c}7.9663 \\
(0.0075)\end{array}$ & $\begin{array}{c}8.0017 \\
(0.0269)\end{array}$ & $\begin{array}{c}8.0057 \\
(0.0067)\end{array}$ & $\begin{array}{l}11.9832 \\
(0.0454)\end{array}$ & $\begin{array}{l}11.9892 \\
(0.0113)\end{array}$ & $\begin{array}{l}12.1226 \\
(0.0511)\end{array}$ & $\begin{array}{l}12.1287 \\
(0.0127)\end{array}$ & $\begin{array}{c}0.4777 \\
(0.0050)\end{array}$ & $\begin{array}{c}0.4780 \\
(0.0012)\end{array}$ & $\begin{array}{c}0.6321 \\
(0.0065)\end{array}$ & $\begin{array}{l}0.6325 \\
(0.0016)\end{array}$ \\
\hline
\end{tabular}

Table 9. B.Es and P.Rs under Exponential Prior

\begin{tabular}{|c|c|c|c|c|c|c|c|c|c|c|c|c|}
\hline & SELF & PLF & SELF & PLF & SELF & PLF & SELF & PLF & SELF & PLF & SELF & PLF \\
\hline & \multicolumn{2}{|c|}{$\alpha_{1}=4, p=0.45$} & \multicolumn{2}{|c|}{$a_{1}=4, p=0.60$} & \multicolumn{2}{|c|}{$\alpha_{2}=6, p=0.45$} & \multicolumn{2}{|c|}{$\alpha_{2}=6, p=0.60$} & \multicolumn{2}{|c|}{$p=0.45$} & \multicolumn{2}{|c|}{$p=0.60$} \\
\hline 50 & $\begin{array}{r}4.4724 \\
(0.1939)\end{array}$ & $\begin{array}{c}4.4954 \\
(0.0441)\end{array}$ & $\begin{array}{l}4.3595 \\
(0.1890)\end{array}$ & $\begin{array}{l}4.3820 \\
(0.0430)\end{array}$ & $\begin{array}{c}6.7310 \\
(0.2918)\end{array}$ & $\begin{array}{c}6.7656 \\
(0.0664)\end{array}$ & $\begin{array}{c}6.6701 \\
(0.2891)\end{array}$ & $\begin{array}{c}6.7044 \\
(0.0658)\end{array}$ & $\begin{array}{c}0.5143 \\
(0.0520)\end{array}$ & $\begin{array}{c}0.5170 \\
(0.0118)\end{array}$ & $\begin{array}{c}0.6648 \\
(0.0719)\end{array}$ & $\begin{array}{c}0.6682 \\
(0.0164)\end{array}$ \\
\hline 100 & $\begin{array}{c}4.4391 \\
(0.0940)\end{array}$ & $\begin{array}{c}4.4504 \\
(0.0216)\end{array}$ & $\begin{array}{c}4.3271 \\
(0.0917)\end{array}$ & $\begin{array}{c}4.3381 \\
(0.0211)\end{array}$ & $\begin{array}{c}6.6809 \\
(0.1415)\end{array}$ & $\begin{array}{c}6.6978 \\
(0.0325)\end{array}$ & $\begin{array}{c}6.6204 \\
(0.1403)\end{array}$ & $\begin{array}{c}6.6372 \\
(0.0322)\end{array}$ & $\begin{array}{c}0.5105 \\
(0.0252)\end{array}$ & $\begin{array}{c}0.5118 \\
(0.0058)\end{array}$ & $\begin{array}{c}0.6599 \\
(0.0349)\end{array}$ & $\begin{array}{c}0.6616 \\
(0.0080)\end{array}$ \\
\hline 200 & $\begin{array}{l}4.3540 \\
(0.0449)\end{array}$ & $\begin{array}{l}4.3595 \\
(0.0105)\end{array}$ & $\begin{array}{c}4.2441 \\
(0.0438)\end{array}$ & $\begin{array}{l}4.2495 \\
(0.0103)\end{array}$ & $\begin{array}{c}6.5528 \\
(0.0676)\end{array}$ & $\begin{array}{c}6.5611 \\
(0.0158)\end{array}$ & $\begin{array}{r}6.4935 \\
(0.0670)\end{array}$ & $\begin{array}{c}6.5017 \\
(0.0157)\end{array}$ & $\begin{array}{c}0.5007 \\
(0.0121)\end{array}$ & $\begin{array}{c}0.5013 \\
(0.0028)\end{array}$ & $\begin{array}{c}0.6472 \\
(0.0166)\end{array}$ & $\begin{array}{l}0.6480 \\
(0.0039)\end{array}$ \\
\hline 300 & $\begin{array}{l}4.2322 \\
(0.0282)\end{array}$ & $\begin{array}{l}4.2357 \\
(0.0068)\end{array}$ & $\begin{array}{c}4.1253 \\
(0.0275)\end{array}$ & $\begin{array}{l}4.1288 \\
(0.0066)\end{array}$ & $\begin{array}{c}6.3694 \\
(0.0425)\end{array}$ & $\begin{array}{c}6.3748 \\
(0.0102)\end{array}$ & $\begin{array}{c}6.3118 \\
(0.0421)\end{array}$ & $\begin{array}{c}6.3171 \\
(0.0101)\end{array}$ & $\begin{array}{l}0.4867 \\
(0.0076)\end{array}$ & $\begin{array}{c}0.4871 \\
(0.0018)\end{array}$ & $\begin{array}{c}0.6291 \\
(0.0105)\end{array}$ & $\begin{array}{l}0.6296 \\
(0.0025)\end{array}$ \\
\hline 400 & $\begin{array}{l}4.1332 \\
(0.0202)\end{array}$ & $\begin{array}{l}4.1358 \\
(0.0050)\end{array}$ & $\begin{array}{l}4.0289 \\
(0.0196)\end{array}$ & $\begin{array}{l}4.0314 \\
(0.0048)\end{array}$ & $\begin{array}{c}6.2205 \\
(0.0303)\end{array}$ & $\begin{array}{c}6.2244 \\
(0.0075)\end{array}$ & $\begin{array}{c}6.1642 \\
(0.0301)\end{array}$ & $\begin{array}{c}6.1680 \\
(0.0074)\end{array}$ & $\begin{array}{c}0.4753 \\
(0.0054)\end{array}$ & $\begin{array}{c}0.4756 \\
(0.0013)\end{array}$ & $\begin{array}{c}0.6144 \\
(0.0075)\end{array}$ & $\begin{array}{l}0.6148 \\
(0.0018)\end{array}$ \\
\hline 500 & $\begin{array}{r}4.1055 \\
(0.0159)\end{array}$ & $\begin{array}{l}4.1076 \\
(0.0040)\end{array}$ & $\begin{array}{c}4.0019 \\
(0.0155)\end{array}$ & $\begin{array}{r}4.0039 \\
(0.0039)\end{array}$ & $\begin{array}{c}6.1788 \\
(0.0239)\end{array}$ & $\begin{array}{c}6.1819 \\
(0.0059)\end{array}$ & $\begin{array}{c}6.1229 \\
(0.0237)\end{array}$ & $\begin{array}{c}6.1260 \\
(0.0059)\end{array}$ & $\begin{array}{l}0.4721 \\
(0.0043)\end{array}$ & $\begin{array}{c}0.4724 \\
(0.0011)\end{array}$ & $\begin{array}{c}0.6103 \\
(0.0059)\end{array}$ & $\begin{array}{l}0.6106 \\
(0.0015)\end{array}$ \\
\hline
\end{tabular}


ON PREDICTIONS FOR MIXTURE OF THE GOMPERTZ DISTRIBUTION

Table 10. B.Es and P.Rs under Exponential Prior

\begin{tabular}{|c|c|c|c|c|c|c|c|c|c|c|c|c|}
\hline & SELF & PLF & SELF & PLF & SELF & PLF & SELF & PLF & SELF & PLF & SELF & PLF \\
\hline & \multicolumn{2}{|c|}{$a_{1}=8, p=0.45$} & \multicolumn{2}{|c|}{$a_{1}=8, p=0.60$} & \multicolumn{2}{|c|}{$\alpha_{2}=12, p=0.45$} & \multicolumn{2}{|c|}{$\alpha_{2}=12, p=0.60$} & \multicolumn{2}{|c|}{$p=0.45$} & \multicolumn{2}{|c|}{$p=0.60$} \\
\hline 50 & $\begin{array}{c}8.7712 \\
(0.3802)\end{array}$ & $\begin{array}{c}8.8163 \\
(0.0866)\end{array}$ & $\begin{array}{c}8.8146 \\
(0.3388)\end{array}$ & $\begin{array}{c}8.8599 \\
(0.0771)\end{array}$ & $\begin{array}{l}13.2006 \\
(0.5722)\end{array}$ & $\begin{array}{l}13.2685 \\
(0.1303)\end{array}$ & $\begin{array}{l}13.3541 \\
(0.6437)\end{array}$ & $\begin{array}{l}13.4228 \\
(0.1465)\end{array}$ & $\begin{array}{c}0.5263 \\
(0.0646)\end{array}$ & $\begin{array}{c}0.5290 \\
(0.0147)\end{array}$ & $\begin{array}{c}0.6964 \\
(0.0846)\end{array}$ & $\begin{array}{r}0.6999 \\
(0.0193)\end{array}$ \\
\hline 100 & $\begin{array}{c}8.7059 \\
(0.1844)\end{array}$ & $\begin{array}{c}8.7280 \\
(0.0424)\end{array}$ & $\begin{array}{c}8.7490 \\
(0.1643)\end{array}$ & $\begin{array}{c}8.7712 \\
(0.0377)\end{array}$ & $\begin{array}{l}13.1024 \\
(0.2776)\end{array}$ & $\begin{array}{l}13.1356 \\
(0.0637)\end{array}$ & $\begin{array}{l}13.2547 \\
(0.3123)\end{array}$ & $\begin{array}{l}13.2883 \\
(0.0717)\end{array}$ & $\begin{array}{c}0.5224 \\
(0.0314)\end{array}$ & $\begin{array}{c}0.5237 \\
(0.0072)\end{array}$ & $\begin{array}{c}0.6912 \\
(0.0410)\end{array}$ & $\begin{array}{r}0.6929 \\
(0.0094)\end{array}$ \\
\hline 200 & $\begin{array}{c}8.5390 \\
(0.0880)\end{array}$ & $\begin{array}{c}8.5498 \\
(0.0206)\end{array}$ & $\begin{array}{c}8.5813 \\
(0.0785)\end{array}$ & $\begin{array}{c}8.5921 \\
(0.0184)\end{array}$ & $\begin{array}{l}12.8512 \\
(0.1325)\end{array}$ & $\begin{array}{l}12.8674 \\
(0.0310)\end{array}$ & $\begin{array}{l}13.0006 \\
(0.1491)\end{array}$ & $\begin{array}{l}13.0170 \\
(0.0349)\end{array}$ & $\begin{array}{c}0.5123 \\
(0.0150)\end{array}$ & $\begin{array}{c}0.5130 \\
(0.0035)\end{array}$ & $\begin{array}{c}0.6779 \\
(0.0196)\end{array}$ & $\begin{array}{r}0.6788 \\
(0.0046)\end{array}$ \\
\hline 300 & $\begin{array}{c}8.3000 \\
(0.0553)\end{array}$ & $\begin{array}{c}8.3070 \\
(0.0133)\end{array}$ & $\begin{array}{c}8.3411 \\
(0.0493)\end{array}$ & $\begin{array}{l}8.3481 \\
(0.0119)\end{array}$ & $\begin{array}{l}12.4915 \\
(0.0833)\end{array}$ & $\begin{array}{l}12.5020 \\
(0.0201)\end{array}$ & $\begin{array}{l}12.6368 \\
(0.0937)\end{array}$ & $\begin{array}{l}12.6473 \\
(0.0226)\end{array}$ & $\begin{array}{r}0.4980 \\
(0.0094)\end{array}$ & $\begin{array}{c}0.4984 \\
(0.0023)\end{array}$ & $\begin{array}{l}0.6589 \\
(0.0123)\end{array}$ & $\begin{array}{c}0.6595 \\
(0.0030)\end{array}$ \\
\hline 400 & $\begin{array}{c}8.1059 \\
(0.0395)\end{array}$ & $\begin{array}{c}8.1110 \\
(0.0098)\end{array}$ & $\begin{array}{c}8.1460 \\
(0.0352)\end{array}$ & $\begin{array}{c}8.1511 \\
(0.0087)\end{array}$ & $\begin{array}{l}12.1994 \\
(0.0595)\end{array}$ & $\begin{array}{l}12.2070 \\
(0.0147)\end{array}$ & $\begin{array}{l}12.3412 \\
(0.0669)\end{array}$ & $\begin{array}{l}12.3490 \\
(0.0165)\end{array}$ & $\begin{array}{c}0.4864 \\
(0.0067)\end{array}$ & $\begin{array}{c}0.4867 \\
(0.0017)\end{array}$ & $\begin{array}{c}0.6435 \\
(0.0088)\end{array}$ & $\begin{array}{r}0.6439 \\
(0.0022)\end{array}$ \\
\hline 500 & $\begin{array}{c}8.0517 \\
(0.0312)\end{array}$ & $\begin{array}{c}8.0557 \\
(0.0077)\end{array}$ & $\begin{array}{l}8.0915 \\
(0.0278)\end{array}$ & $\begin{array}{l}8.0956 \\
(0.0069)\end{array}$ & $\begin{array}{l}12.1177 \\
(0.0469)\end{array}$ & $\begin{array}{l}12.1238 \\
(0.0117)\end{array}$ & $\begin{array}{l}12.2586 \\
(0.0528)\end{array}$ & $\begin{array}{l}12.2648 \\
(0.0131)\end{array}$ & $\begin{array}{l}0.4831 \\
(0.0053)\end{array}$ & $\begin{array}{c}0.4833 \\
(0.0013)\end{array}$ & $\begin{array}{c}0.6392 \\
(0.0069)\end{array}$ & $\begin{array}{c}0.6396 \\
(0.0017)\end{array}$ \\
\hline
\end{tabular}

Table 11. 95\% credible intervals under Uniform Prior

\begin{tabular}{|c|c|c|c|c|c|c|c|c|c|}
\hline \multirow{2}{*}{$n$} & \multicolumn{3}{|c|}{$\alpha_{1}=4$} & \multicolumn{3}{|c|}{$a_{2}=6$} & \multicolumn{3}{|c|}{$p=0.45$} \\
\hline & $L L$ & $U L$ & $U L-L L$ & $L L$ & $U L$ & $U L-L L$ & $L L$ & $U L$ & $U L-L L$ \\
\hline 50 & 3.4241 & 4.9498 & 1.5257 & 5.1532 & 7.4494 & 2.2962 & 0.3938 & 0.5692 & 0.1755 \\
\hline 100 & 3.4882 & 4.9129 & 1.4248 & 5.2497 & 7.3939 & 2.1443 & 0.4011 & 0.5650 & 0.1638 \\
\hline 200 & 3.5092 & 4.8187 & 1.3096 & 5.2813 & 7.2522 & 1.9709 & 0.4036 & 0.5542 & 0.1506 \\
\hline 300 & 3.5391 & 4.6839 & 1.1448 & 5.3263 & 7.0492 & 1.7229 & 0.4070 & 0.5386 & 0.1317 \\
\hline 400 & 3.6231 & 4.5743 & 0.9512 & 5.4528 & 6.8844 & 1.4316 & 0.4167 & 0.5260 & 0.1094 \\
\hline 500 & 3.6817 & 4.5437 & 0.8620 & 5.5410 & 6.8383 & 1.2973 & 0.4234 & 0.5225 & 0.0991 \\
\hline
\end{tabular}

Table 12. 95\% credible intervals under Jeffreys Prior

\begin{tabular}{|c|c|c|c|c|c|c|c|c|c|}
\hline \multirow{2}{*}{$n$} & \multicolumn{3}{|c|}{$a_{1}=4$} & \multicolumn{3}{|c|}{$\alpha_{2}=6$} & \multicolumn{3}{|c|}{$p=0.45$} \\
\hline & $L L$ & $U L$ & $U L-L L$ & $L L$ & $U L$ & $U L-L L$ & $L L$ & $U L$ & $U L-L L$ \\
\hline 50 & 3.3898 & 4.9003 & 1.5104 & 5.1017 & 7.3749 & 2.2732 & 0.3898 & 0.5635 & 0.1737 \\
\hline 100 & 3.4533 & 4.8638 & 1.4105 & 5.1972 & 7.3200 & 2.1228 & 0.3971 & 0.5593 & 0.1622 \\
\hline 200 & 3.4741 & 4.7705 & 1.2965 & 5.2285 & 7.1797 & 1.9512 & 0.3995 & 0.5486 & 0.1491 \\
\hline 300 & 3.5037 & 4.6370 & 1.1334 & 5.2730 & 6.9787 & 1.7057 & 0.4029 & 0.5333 & 0.1303 \\
\hline 400 & 3.5869 & 4.5286 & 0.9417 & 5.3983 & 6.8155 & 1.4172 & 0.4125 & 0.5208 & 0.1083 \\
\hline 500 & 3.6449 & 4.4983 & 0.8534 & 5.4856 & 6.7699 & 1.2843 & 0.4192 & 0.5173 & 0.0981 \\
\hline
\end{tabular}




\section{FEROZE \& ASLAM}

Table 13. 95\% credible intervals under Gamma Prior

\begin{tabular}{|c|c|c|c|c|c|c|c|c|c|}
\hline \multirow{2}{*}{$n$} & \multicolumn{3}{|c|}{$a_{1}=4$} & \multicolumn{3}{|c|}{$\alpha_{2}=6$} & \multicolumn{3}{|c|}{$p=0.45$} \\
\hline & $L L$ & $U L$ & $U L-L L$ & $L L$ & $U L$ & $U L-L L$ & $L L$ & $U L$ & $U L-L L$ \\
\hline 50 & 3.3213 & 4.8013 & 1.4799 & 4.9986 & 7.2259 & 2.2273 & 0.3820 & 0.5521 & 0.1702 \\
\hline 100 & 3.3835 & 4.7655 & 1.3820 & 5.0922 & 7.1721 & 2.0799 & 0.3891 & 0.5480 & 0.1589 \\
\hline 200 & 3.4039 & 4.6742 & 1.2703 & 5.1228 & 7.0346 & 1.9118 & 0.3914 & 0.5375 & 0.1461 \\
\hline 300 & 3.4329 & 4.5433 & 1.1105 & 5.1665 & 6.8377 & 1.6712 & 0.3948 & 0.5225 & 0.1277 \\
\hline 400 & 3.5144 & 4.4371 & 0.9227 & 5.2892 & 6.6778 & 1.3886 & 0.4042 & 0.5103 & 0.1061 \\
\hline 500 & 3.5713 & 4.4074 & 0.8361 & 5.3748 & 6.6331 & 1.2584 & 0.4107 & 0.5069 & 0.0962 \\
\hline
\end{tabular}

Table 14. 95\% credible intervals under Chi Square Prior

\begin{tabular}{|c|c|c|c|c|c|c|c|c|c|}
\hline \multirow{2}{*}{$n$} & \multicolumn{3}{|c|}{$a_{1}=4$} & \multicolumn{3}{|c|}{$\alpha_{2}=6$} & \multicolumn{3}{|c|}{$p=0.45$} \\
\hline & $L L$ & $U L$ & $U L-L L$ & $L L$ & $U L$ & $U L-L L$ & $L L$ & $U L$ & $U L-L L$ \\
\hline 50 & 3.3556 & 4.8508 & 1.4952 & 5.0501 & 7.3004 & 2.2503 & 0.3859 & 0.5578 & 0.1719 \\
\hline 100 & 3.4184 & 4.8146 & 1.3963 & 5.1447 & 7.2460 & 2.1014 & 0.3931 & 0.5537 & 0.1606 \\
\hline 200 & 3.4390 & 4.7224 & 1.2834 & 5.1757 & 7.1071 & 1.9315 & 0.3955 & 0.5431 & 0.1476 \\
\hline 300 & 3.4683 & 4.5902 & 1.1219 & 5.2198 & 6.9082 & 1.6885 & 0.3989 & 0.5279 & 0.1290 \\
\hline 400 & 3.5507 & 4.4828 & 0.9322 & 5.3438 & 6.7467 & 1.4029 & 0.4083 & 0.5155 & 0.1072 \\
\hline 500 & 3.6081 & 4.4528 & 0.8447 & 5.4302 & 6.7015 & 1.2713 & 0.4149 & 0.5121 & 0.0971 \\
\hline
\end{tabular}

Table 15. 95\% credible intervals under Exponential Prior

\begin{tabular}{|c|c|c|c|c|c|c|c|c|c|}
\hline \multirow{2}{*}{$n$} & \multicolumn{3}{|c|}{$a_{1}=4$} & \multicolumn{3}{|c|}{$\alpha_{2}=6$} & \multicolumn{3}{|c|}{$p=0.45$} \\
\hline & $L L$ & UL & $U L-L L$ & $L L$ & $U L$ & $U L-L L$ & $L L$ & UL & $U L-L L$ \\
\hline 50 & 3.3932 & 4.9052 & 1.5120 & 5.1068 & 7.3823 & 2.2755 & 0.3902 & 0.5641 & 0.1739 \\
\hline 100 & 3.4568 & 4.8687 & 1.4119 & 5.2024 & 7.3274 & 2.1250 & 0.3975 & 0.5599 & 0.1624 \\
\hline 200 & 3.4776 & 4.7754 & 1.2978 & 5.2338 & 7.1869 & 1.9532 & 0.3999 & 0.5492 & 0.1492 \\
\hline 300 & 3.5072 & 4.6417 & 1.1345 & 5.2783 & 6.9858 & 1.7074 & 0.4033 & 0.5338 & 0.1305 \\
\hline 400 & 3.5905 & 4.5332 & 0.9426 & 5.4037 & 6.8224 & 1.4187 & 0.4129 & 0.5213 & 0.1084 \\
\hline 500 & 3.6486 & 4.5028 & 0.8542 & 5.4911 & 6.7767 & 1.2856 & 0.4196 & 0.5178 & 0.0982 \\
\hline
\end{tabular}


ON PREDICTIONS FOR MIXTURE OF THE GOMPERTZ DISTRIBUTION

Table 16. 95\% posterior predictive intervals under different priors

\begin{tabular}{ccccccc}
\hline $\mathbf{n}$ & Limits & Uniform & Jeffreys & Gamma & Chi square & Exponential \\
\hline \multirow{2}{*}{50} & LL & 2.4773 & 2.4037 & 2.2609 & 2.3316 & 2.4110 \\
& UL & 17.9778 & 17.4438 & 16.4078 & 16.9206 & 17.4967 \\
& UL - LL & 15.5005 & 15.0401 & 14.1469 & 14.5890 & 15.0858 \\
\hline \multirow{2}{*}{$\mathbf{1 0 0}$} & LL & 2.6190 & 2.5412 & 2.3903 & 2.4650 & 2.5489 \\
& UL & 17.5793 & 17.0572 & 16.0442 & 16.5455 & 17.1089 \\
& UL - LL & 14.9603 & 14.5160 & 13.6539 & 14.0805 & 14.5600 \\
\hline \multirow{2}{*}{$\mathbf{2 0 0}$} & LL & 2.6666 & 2.5874 & 2.4338 & 2.5098 & 2.5953 \\
& UL & 16.5876 & 16.0950 & 15.1391 & 15.6121 & 16.1438 \\
& UL - LL & 13.9210 & 13.5075 & 12.7053 & 13.1023 & 13.5485 \\
\hline \multirow{3}{*}{$\mathbf{3 0 0}$} & LL & 2.7354 & 2.6541 & 2.4965 & 2.5745 & 2.6622 \\
& UL & 15.2334 & 14.7810 & 13.9031 & 14.3376 & 14.8258 \\
& UL - LL & 12.4981 & 12.1269 & 11.4066 & 11.7631 & 12.1636 \\
\hline \multirow{2}{*}{$\mathbf{4 0 0}$} & LL & 2.9350 & 2.8478 & 2.6787 & 2.7624 & 2.8564 \\
& UL & 14.1895 & 13.7681 & 12.9504 & 13.3551 & 13.8098 \\
& UL - LL & 11.2546 & 10.9203 & 10.2717 & 10.5927 & 10.9534 \\
\hline \multirow{2}{*}{$\mathbf{5 0 0}$} & LL & 3.0797 & 2.9882 & 2.8108 & 2.8986 & 2.9973 \\
& UL & 13.9065 & 13.4935 & 12.6921 & 13.0887 & 13.5344 \\
& UL - LL & 10.8268 & 10.5052 & 9.8813 & 10.1901 & 10.5371 \\
\hline
\end{tabular}

The simulation study has been conducted under the assumption of different priors using two loss functions. The performance of different estimators has been compared in terms of posterior risks and rate of convergence. It has been observed that the estimated value of the parameter converges to the true value of the parameter by increasing the sample size. This pattern is similar under each prior and for every loss function. In cases of non-informative priors, the estimates under the Jeffreys prior provide better convergence, while among informative priors the gamma prior gives comparatively rapid convergence. On the other hand, use of squared error loss function results in faster convergence than precautionary loss function. It is interesting to note that for each combination of prior and loss function, the increased values of the parameters impose a negative impact on the convergence rate of the estimates. However, increasing the value of weight parameter has a positive effect on the convergence of the corresponding estimators but convergence rate of the weight estimator itself becomes slower. All the parameters have been overestimated in almost all the cases and the extent of 
overestimation is greater for larger true parametric values. On the whole, it can be assessed that the estimates under gamma prior using precautionary loss function have the best convergence rate. Some prior elicitation technique may further strengthen this argument.

It is apparent from the above tables that the magnitude of posterior risks is indirectly proportional to sample size, while it is directly related to the true value of the parameter. This property holds for all estimators. The estimates under the Jeffreys prior have smaller risks than those under uniform prior. Similarly, among all informative priors, the estimates using gamma prior are associated with the minimum risks. In a comparison of informative and non-informative priors, informative priors perform better. This indicates the dominance of informative priors over non-informative priors. The performance of the estimates (representing the corresponding component) has been positively affected by increasing the values of the weight parameter, but at the cost of inflated risks for the parameter itself. It can also be observed that the estimates under precautionary loss function have smaller risks than those based on squared error loss function, irrespective of choice of prior and the true parametric values. Therefore, in terms of posterior risks, the estimates using gamma prior based on precautionary loss function provide the best point estimation.

In case of interval estimation (presented in Tables 11-16), the widths of 95\% credible intervals decrease when increasing the sample size. The least amount of widths for credible intervals has been observed under gamma prior. In addition, the posterior predictions tend to be more specific under gamma prior. This is another indication that the gamma prior performs well as compared to other priors.

\section{Real Life Example}

Real life data regarding cancer survival times in years presented by Bekker et al. (2000) has been analyzed to illustrate the practical applicability of the results. The test termination time is considered to be such that the overall sample is $15 \%$ censored.

The analysis of real life data replicated the patterns observed under simulation study. The point and interval estimates for the parameters of the Gompertz mixture model; based on gamma prior; using PLF are found to be the most efficient. The posterior predictive intervals have the least amounts of widths again under gamma prior. So, in order to estimate the said parameters and to make predictions of the future values of the variable from the mentioned model, the use of gamma prior under PLF may be preferred. 
ON PREDICTIONS FOR MIXTURE OF THE GOMPERTZ DISTRIBUTION

Table 17. Bayes estimates and posterior risks under real life data

\begin{tabular}{ccccccc}
\hline \multirow{2}{*}{ Priors } & \multicolumn{3}{c}{ SELF } & \multicolumn{3}{c}{$\boldsymbol{P L F}$} \\
\cline { 2 - 7 } & $\boldsymbol{\alpha}_{\mathbf{1}}$ & $\boldsymbol{\alpha}_{\mathbf{2}}$ & $\boldsymbol{p}=\mathbf{0 . 4 5}$ & $\boldsymbol{\alpha}_{\mathbf{1}}$ & $\boldsymbol{\alpha}_{\mathbf{2}}$ & $\boldsymbol{p}=\mathbf{0 . 4 5}$ \\
\hline \multirow{2}{*}{ Uniform } & 2.0841 & 2.3456 & 0.4963 & 2.0948 & 2.3577 & 0.4988 \\
& $(1.1218)$ & $(1.0466)$ & $(0.1298)$ & $(0.2554)$ & $(0.2382)$ & $(0.0296)$ \\
\hline \multirow{2}{*}{ Jefrreys } & 2.0632 & 2.3222 & 0.4913 & 2.0738 & 2.3341 & 0.4939 \\
& $(1.1106)$ & $(1.0361)$ & $(0.1169)$ & $(0.2528)$ & $(0.2359)$ & $(0.0266)$ \\
\hline \multirow{2}{*}{ Gamma } & 2.0215 & 2.2753 & 0.4814 & 2.0319 & 2.2870 & 0.4839 \\
& $(1.0096)$ & $(0.9419)$ & $(0.0909)$ & $(0.2298)$ & $(0.2144)$ & $(0.0207)$ \\
\hline \multirow{2}{*}{ Chi Square } & 2.0424 & 2.2987 & 0.4864 & 2.0529 & 2.3105 & 0.4889 \\
& $(1.0321)$ & $(0.9629)$ & $(0.1039)$ & $(0.2349)$ & $(0.2192)$ & $(0.0236)$ \\
\hline \multirow{2}{*}{ Exponential } & 2.0653 & 2.3245 & 0.4918 & 2.0759 & 2.3365 & 0.4944 \\
& $(1.0668)$ & $(0.9953)$ & $(0.1105)$ & $(0.2429)$ & $(0.2266)$ & $(0.0252)$ \\
\hline
\end{tabular}

Table 18. 95\% credible intervals under real life data

\begin{tabular}{|c|c|c|c|c|c|c|c|c|c|}
\hline \multirow{2}{*}{ Priors } & \multicolumn{3}{|c|}{$\alpha_{1}$} & \multicolumn{3}{|c|}{$\alpha_{2}$} & \multicolumn{3}{|c|}{$p=0.45$} \\
\hline & $L L$ & $U L$ & $U L-L L$ & $L L$ & $U L$ & $U L-L L$ & $L L$ & UL & $U L-L L$ \\
\hline Uniform & 1.7475 & 2.4206 & 0.6731 & 2.0316 & 2.8689 & 0.8373 & 0.3924 & 0.5872 & 0.1948 \\
\hline Jeffreys & 1.7300 & 2.3964 & 0.6664 & 2.0113 & 2.8402 & 0.8289 & 0.3978 & 0.5731 & 0.1753 \\
\hline Gamma & 1.7186 & 2.3244 & 0.6058 & 1.9927 & 2.7462 & 0.7535 & 0.4087 & 0.5450 & 0.1363 \\
\hline Chi Square & 1.7328 & 2.3520 & 0.6192 & 2.0099 & 2.7801 & 0.7703 & 0.4033 & 0.5591 & 0.1558 \\
\hline Exponential & 1.7452 & 2.3854 & 0.6401 & 2.0259 & 2.8222 & 0.7962 & 0.4034 & 0.5692 & 0.1657 \\
\hline
\end{tabular}

Table 19. 95\% posterior predictive intervals under real life data

\begin{tabular}{cccc}
\hline Priors & LL & UL & UL-LL \\
\hline Uniform & 0.6227 & 11.0830 & 10.4602 \\
Jeffreys & 0.6165 & 10.9721 & 10.3556 \\
Gamma & 0.6124 & 10.6426 & 10.0302 \\
Chi Square & 0.6175 & 10.7688 & 10.1514 \\
Exponential & 0.6219 & 10.9216 & 10.2997 \\
\hline
\end{tabular}




\section{Conclusion}

The study proposed the point and interval estimators for the parameters of the two-component mixture of the Gompertz distribution under a Bayesian framework along with posterior predictions for the future value from said model. The performance of the different estimators has been compared in terms of posterior risks (for point estimators) and widths of interval estimates with respect to various priors and loss functions. The findings of the study suggest that for Bayesian estimation of the parameters (along with posterior predictions) of the two-component mixture of the Gompertz distribution, the use of gamma prior under precautionary loss function is preferred. The proposed estimators are consistent in nature. The results of the study are useful for practitioners looking to model some failure time data, where the cases of failures are more than one.

\section{References}

Aslam, M. (2003). An application of prior predictive distribution to elicit the prior density. Journal of Statistical Theory and Applications, 2(1), 183-197.

Bekker, A., Roux, J., \& Mostert, P. (2000). A generalization of the compound Rayleigh distribution: using a Bayesian method on cancer survival times. Communication in Statistics-Theory and Methods, 29(7), 1419-1433.

Eberlya, L. E., \& Casella, G. (2003). Estimating Bayesian credible intervals. Journal of Statistical Planning and Inference, 112, 115-132.

Ismail, A. A. (2010). Bayes estimation of Gompertz distribution parameters and acceleration factor under partially accelerated life tests with type-I censoring. Journal of Statistical Computation and Simulation, 80(11), 1253-1264.

Ismail, A. A. (2011). Planning step-stress life tests with type-II censored Data. Scientific Research and Essays, 6(19), 4021-4028.

Jeffreys, H. (1961). Theory of Probability. 3rd Ed. Oxford: Oxford University Press.

Jaheen, Z. F. (2003). A Bayesian analysis of record statistics from the Gompertz model. Applied Mathematical Computations, 145(2-3), 307-320.

Kazmi, S.M.A., Aslam, M., \& Ali, S. (2012). On the Bayesian estimation for two component mixture of maxwell distribution, assuming type I censored data. International Journal of Applied Science and Technology (IJAST), 2(1), 197-218. 
Khedhairi, A., \& Gohary, A. E. (2008). A new class of bivariate Gompertz distributions and its mixture. International Journal of Mathematical Analysis, 2(5), 235-253.

Kiani, K., Arasan, J., \& Midi, H. (2012). Interval estimations for parameters of Gompertz model with time-dependent covariate and right censored data. Sains Malaysiana, 41(4), 471-480.

Laplace, P. S. (1812). Theorie Analytique Des Probabilities. Veuve Courcier Paris.

Majeed, M.Y., \& Aslam, M. (2012). Bayesian analysis of the two component mixture of inverted exponential distribution under quadratic loss functions. International Journal of Physical Sciences, 7(9), 1424-1434.

Pollard, J., \& Valkovics, E. (1992). The Gompertz distribution and its applications. Genus, 48(3-4), 15-29.

Saleem, M., \& Aslam, M. (2008). Bayesian analysis of the two component mixture of the Rayleigh dist. With the uniform and the Jeffreys priors. Journal of Applied Statistical Science, 16(4), 105-113.

Saleem, M., Aslam, M., \& Economou, P. (2010). On the Bayesian analysis of the mixture of power function distribution using the complete and the censored sample. Journal of Applied Statistics, 37(1), 25-40.

Saracoglu, B., Kaya, M.F. \& Abd-Elfattah, A.M. (2009). Comparison of estimators for stress-strength reliability in the Gompertz case. Hacettepe Journal of Mathematics and Statistics, 38(3), 339-349.

Willemse, W. and Koppelaar, H. (2000). Knowledge elicitation of Gompertz' law of morality, Scandinavian Actuarial Journal, 94, 168-180.

Wu, C. C., Wu, S. F., \& Chan, H. Y. (2006). MLE and the estimated expected test time for the two-parameter Gompertz distribution under progressive censoring with binomial removals. Applied Mathematical Computation, 181(2), 1657-1670.

Wu, J.W., Hung, W.L., \& Tsai, C.H. (2004). Estimation of parameters of the Gompertz distribution using the least squares method. Applied Mathematical Computation, 158(1), 133-147.

Wu, S.J., Chang, C.T., \& Tsai, T.R. (2003). Point and interval estimations for the Gompertz distribution under progressive type-II censoring. Metron International Journal of Statistics, 21(3), 403-418. 